\title{
Kommunikationsprobleme in Reklamationsgesprächen
}

\author{
Reinhard Fiehler / Walther Kindt / Guido Schnieders
}

veröffentlicht im Sammelband:

Gisela Brünner / Reinhard Fiehler / Walther Kindt (Hrsg.):

Angewandte Diskursforschung

Band 1: Grundlagen und Beispielanalysen

Radolfzell: Verlag für Gesprächsforschung 2002, 120 - 154

ISBN 3 - $936656-01$ - 0 (früher: 3 - 531 - $13076-5$ )

Die PDF-Dateien bewahren die ursprünglichen Seitenumbrüche und Fußnoten-Positionen der ersten Auflage des Buches. Alle Zitationen bleiben also gültig. Bei Zeilenumbrüchen und Layout mussten jedoch Veränderungen vorgenommen werden.

\section{Alle Texte erhältlich unter}

www.verlag-gespraechsforschung.de

\author{
Alle Rechte vorbehalten. \\ $\odot$ Verlag für Gesprächsforschung, Dr. Martin Hartung, Radolfzell 2002 \\ Das Werk einschließlich aller seiner Teile ist urheberrechtlich geschützt. Jede Verwertung \\ außerhalb der engen Grenzen des Urheberrechtgesetzes ist ohne Zustimmung des Verlages \\ unzulässig und strafbar. Das gilt insbesondere für Vervielfältigung, Übersetzungen, \\ Mikroverfilmungen und die Einspeicherung und Verarbeitung in elektronischen Systemen.
}




\title{
Kommunikationsprobleme in Reklamationsgesprächen
}

\author{
Reinhard Fiehler/Walther Kindt/Guido Schnieders
}

\section{Zusammenfassung}

Der Beitrag verdeutlicht am Beispiel eines bestimmten Gesprächstyps - Reklamationsgesprächen -, welche Möglichkeiten die angewandte Diskursforschung zu seiner Analyse hat und wie entsprechende Untersuchungsergebnisse für Trainings fruchtbar gemacht werden können. Speziell wird gezeigt, wie die in Reklamationen auftretenden Kommunikationsprobleme den Aufgaben eines zugrundeliegenden Handlungsschemas zugeordnet werden können und welche Möglichkeiten zur sprachlich-kommunikativen Bewältigung dieser Aufgaben bestehen.

\section{Einleitung}

Fast alle Kundlnnen machen im Laufe ihres (Konsumenten-)Lebens Erfahrungen mit Reklamationsgesprächen, und die Erinnerung an solche Gespräche dürfte häufig zwiespältig sein. Auf der anderen Seite schenken Unternehmen der Analyse von Kommunikation sowie der Schulung der kommunikativen Fähigkeiten ihrer Mitarbeiterlnnen zunehmend mehr Aufmerksamkeit. Erstaunlicherweise gilt dies jedoch nicht gleichermaßen für Reklamationsgespräche. Dabei gehört dieser Gesprächstyp sicherlich zu den für das Unternehmensimage besonders wichtigen Formen unternehmensexterner Kommunikation.

Die zwiespältigen Erfahrungen wie die mangelnde Beachtung haben etwas damit zu tun, daß Reklamationsgespräche ein ebenso heikler wie komplexer Kommunikationstyp sind. In ihm mischen sich Elemente sehr verschiedener Kommunikationsformen wie z.B. Beschwerde, Konfliktaustragung, Argumentation und Beratung.

Reklamationen bedeuten für Unternehmen und KundInnen etwas Unterschiedliches. Sie sind für beide Parteien mit differierenden Interessen verknüpft und in ganz verschiedene Abläufe eingebunden. Hieraus ergibt sich, daß beide Seiten auch unterschiedliche Perspektiven auf Reklamationen haben: Aus Sicht der Unternehmen ist das Auftreten von Reklamationen eine Störung des normalen Geschäftsverlaufs. Viele Unternehmen haben deshalb zu ihrer Bearbeitung, die häufig recht zeit- und arbeitsintensiv ist, eigene Abteilungen eingerichtet und sie so aus dem Prozeß der 'eigentlichen' Leistungserstellung herausgenommen. Reklamationen sind für Unternehmen zugleich aber auch eine Möglichkeit, Informationen über möglicherweise systematische - Produktmängel zu bekommen, und sie werden von vielen Unternehmen auch entsprechend als Marketinginstrument genutzt (vgl. Riemer 1986).

Entsprechen Waren oder Dienstleistungen nicht den Erwartungen, so ist dies auch aus Sicht der Kundlnnen eine Störung der Abläufe des normalen Alltags. Sie ist häufig mit negativen Emotionen verbunden (Enttäuschung, Ärger, Wut etc.). Die Behebung der Störung ist für den Kunden zeit- und arbeitsaufwendig und mit nicht vorhergesehenen Aktivitäten und Kosten verbunden, was die Emotionalität noch verstärken kann. Der Kunde muß sich mit der Reklamation exponieren (aus seiner Kundenanonymität hervortreten) und sich gegen eine größere und möglicherweise überlegene Institution durchsetzen, für die diese Reklamation selbst eine Störung darstellt und der sie deshalb nicht unbedingt positiv gegenübersteht. Auf diesem Hintergrund unterbleibt ein beachtlicher Teil möglicher Reklamationen. 
Wir möchten im folgenden Abschnitt 2 ein authentisches Reklamationsgespräch vorstellen und daran zeigen, $\mathrm{da} ß$ in ihm vielfältige Kommunikationsprobleme auftreten, daß es aber oft nicht einfach ist, solche Probleme genau zu lokalisieren und ihre Ursachen zu benennen. Abschnitt 3 charakterisiert die Besonderheiten des Gesprächstyps "Reklamation" und stellt das analytische Instrumentarium der Diskursforschung vor. Mit seiner Hilfe wird ein klareres Bild und eine genauere Einordnung der Probleme im eingangs diskutierten Transkript gewonnen. Abschnitt 4 gibt dann einen Überblick über typische Kommunikationsprobleme in Reklamationsgesprächen. Sie sind Resultat der diskursanalytischen Untersuchung größerer Korpora von Reklamationen. Zugleich wird an Ausschnitten aus weiteren Reklamationsgesprächen dargestellt, wie Kommunikationsteilnehmer mit einigen dieser typischen Probleme umgehen. D.h. es werden jeweils Positiv- und Negativbeispiele dafür angegeben, wie die Beteiligten solche Probleme lösen oder warum sie an einer Lösung scheitern. Zum Schluß des Beitrags in Abschnitt 5 ziehen wir ein kurzes Fazit aus den dargestellten Ergebnissen und formulieren Konsequenzen für den Inhalt und die Durchführung von Kommunikationstrainings, die sich mit Reklamationsgesprächen befassen.

\section{Ein authentisches Reklamationsgespräch}

Das telefonische Reklamationsgespräch, das wir in diesem Abschnitt vorstellen, entstammt einem größeren Korpus von Reklamationen. Es ist unseren Erfahrungen nach in einem grundsätzlichen Sinne typisch für diese: Es mündet in eine erfolgreiche Problembearbeitung und droht in keiner Phase dramatisch zu scheitern; insofern erfüllt es letztendlich seinen Zweck. Wie wir anhand des größeren Korpus feststellen konnten, gilt das für die meisten Reklamationsgespräche: Spektakuläre Phänomene wie Beschimpfungen oder vorzeitige Gesprächsabbrüche kommen nur in Ausnahmefällen vor. Die Leistung linguistischer Analysen kann aber gerade an solchen eher unauffälligen und alltäglichen Beispielen gezeigt werden.

Unser Beispielgespräch entstammt der Serviceabteilung eines deutschen Unternehmens, das im Direktvertrieb, also ohne Einzelhandel, arbeitet. Bestellungen werden schriftlich oder - im Fall kleinerer Rechnungssummen - telefonisch entgegengenommen; geliefert wird per Post. In einigen größeren Städten unterhält das Unternehmen Filialen, die vor allem Serviceaufgaben (wie Kundenberatungen und Reparaturen) wahrnehmen, bei denen man aber auch Bestellungen aufgeben kann; ansonsten ist dafür die Zentrale zuständig. Das vorliegende Gespräch wird mit der Zentrale geführt; sein Gegenstand - der reklamierte Sachverhalt - ist die mehrfache falsche Ausführung einer Bestellung, die die Kundin bei der örtlichen Filiale telefonisch in Auftrag gegeben hat. Da die Kundin in dieser Angelegenheit schon einmal mit der Zentrale telefoniert hat, stellt das Gespräch nur einen Ausschnitt aus einem komplexeren Reklamationsprozeß dar.

Das folgende Transkript wurde mit dem Programm HIAT-DOS 2.2 angefertigt (zu den Transkriptionskonventionen s. Ehlich/Rehbein 1976). "Tm" bezeichnet den Sachbearbeiter, "Kw" die Kundin. Sämtliche Personen-, Firmen- und Ortsnamen sowie Zahlenangaben sind durch Aliasnamen bzw. andere Zahlen ersetzt; die Größenordnungen von Geldbeträgen blieben dabei in etwa erhalten. Produktbezeichnungen sind im Transkript durch PRODUKT, PRODUKTNAME o.ä. wiedergegeben. 


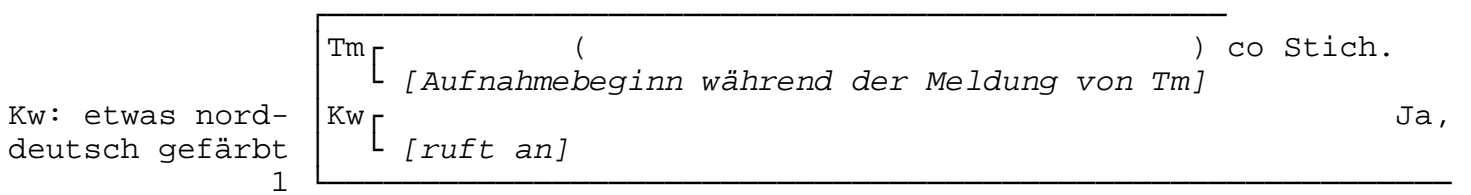
2

Kw [ hier is Saal, guten Tag, ich hatte mich letzte Woche bei

Kw [ Ihnen beschwert über den Osnabrücker Kundendienst, und

Kw [ zwar hatte ich ne .. äh, [PROD]/ also ne [PRODUKTTEIL 1]

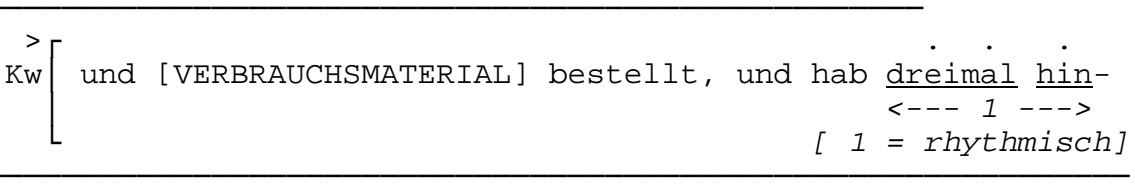

Kw [ tereinander den. äh, [PRODUKTTEIL 2] bekommen. Nun hat-

Kw [ te ich mich bei Ihnen beschwert dadrüber, und hatte das 7

$>/$
$\operatorname{Tm}[\quad$ Hm
Kw [ bei Ihnen bestellt, nun sch/ äh, schicken sie mir n

Kw [ Schreiben, Sie möchten die Auftragsnummer haben. Ich hab

10

Kw [ ja gar keine Auftragsnummer, ich hab das immer telefo-

$\operatorname{Tm}[((3$ Sek.) ) (Schnaufen) Ich kann mich dran Kw [ nisch bestellt.

11

Tm[ erinnern, das Schreiben hab ich letzte Woche gehabt.

$\mathrm{KW}[\mathrm{Ja}$, 12

Kw [ das is ja ärgerlich, daß man nun, das sind jetzt vier

13

Kw [ Wochen, ich denk Sie schicken mir das umgehend zu, und. $\operatorname{Tm}[$ (We/) Ja, wir. können hier 5 Kw[ nun krieg ich wieder $\mathrm{n}$ Schreiben von Ihnen.

Tm[ keine Auftragsnummer ermitteln, wenn hier bei mir jemand 
$\operatorname{Tm}[\quad \underline{J} a \underline{a}$, sagen se mir mal eben mal die Kw [ des vierenvierzig Mark und nich/

Tm[ Nummer aus dem Schreiben, was ich Ihnen jetzt geschickt

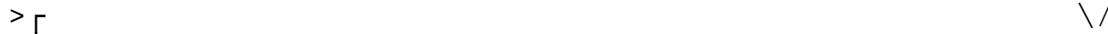

$\operatorname{Tm}[$ habe. $S$ is ne sechsundsechziger Nummer. Ja

$\mathrm{Kw}[\quad$ Ja, sechsundsechzig

Tm meint fünf, sagt aber vier.

${ }_{\operatorname{Tm}}^{>}[/$
Kw [ neun acht, fünf, vier sieben drei. Vier, vier, sieben,

33

$\operatorname{Tm}[$ drei. ((Einatmen))

Kw[ Das is ja $\mathrm{n}$ Unding, die Sachen sind

34

Kw [ so teuer, und und, man sitzt hier damit zu, das muß doch

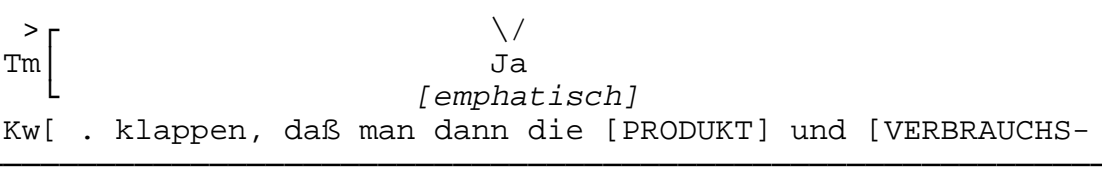

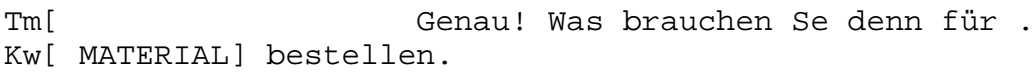

$\operatorname{Tm}[$ [VERBRAUCHSMATERIAL], Frau Saal.

Kw [ Die f/ für den [PRODUKT 38

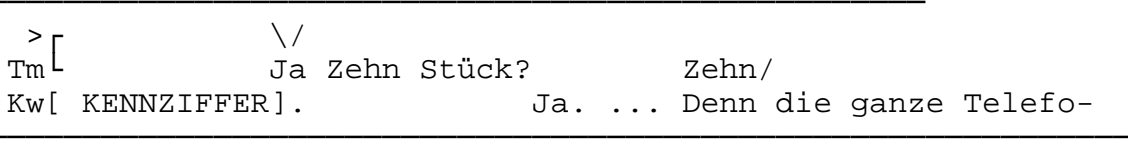

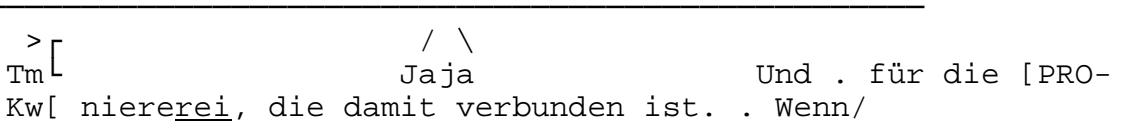

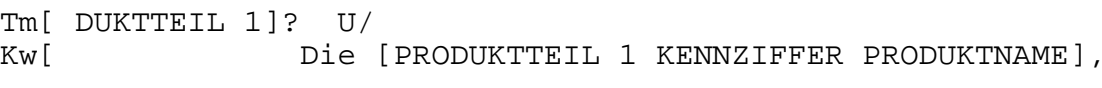

41

$\operatorname{Tm}[\quad$ Die [PRODUKTTEIL], die

Kw [ auch für den [PRODUKT KENNZIFFER].

42

$\operatorname{Tm}_{\operatorname{Tm}[\text { [PRODUKTTEIL }], \text { ne }}^{>}$.. Ja, diese ganze [PRODUKT]! Die ganze




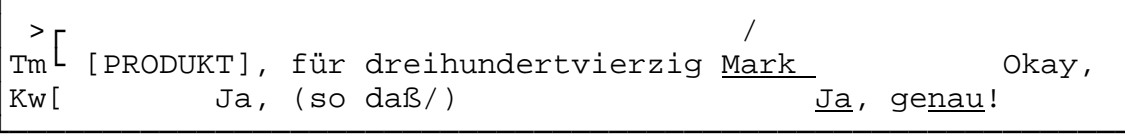

45

Tm[ da gibts aber nur noch die [KENNZIFFER], das ist das

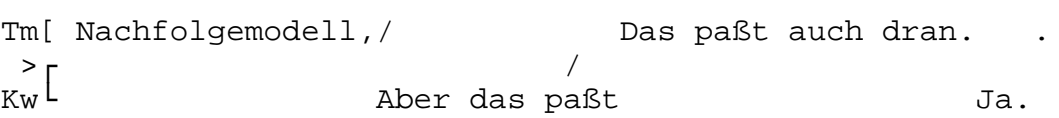




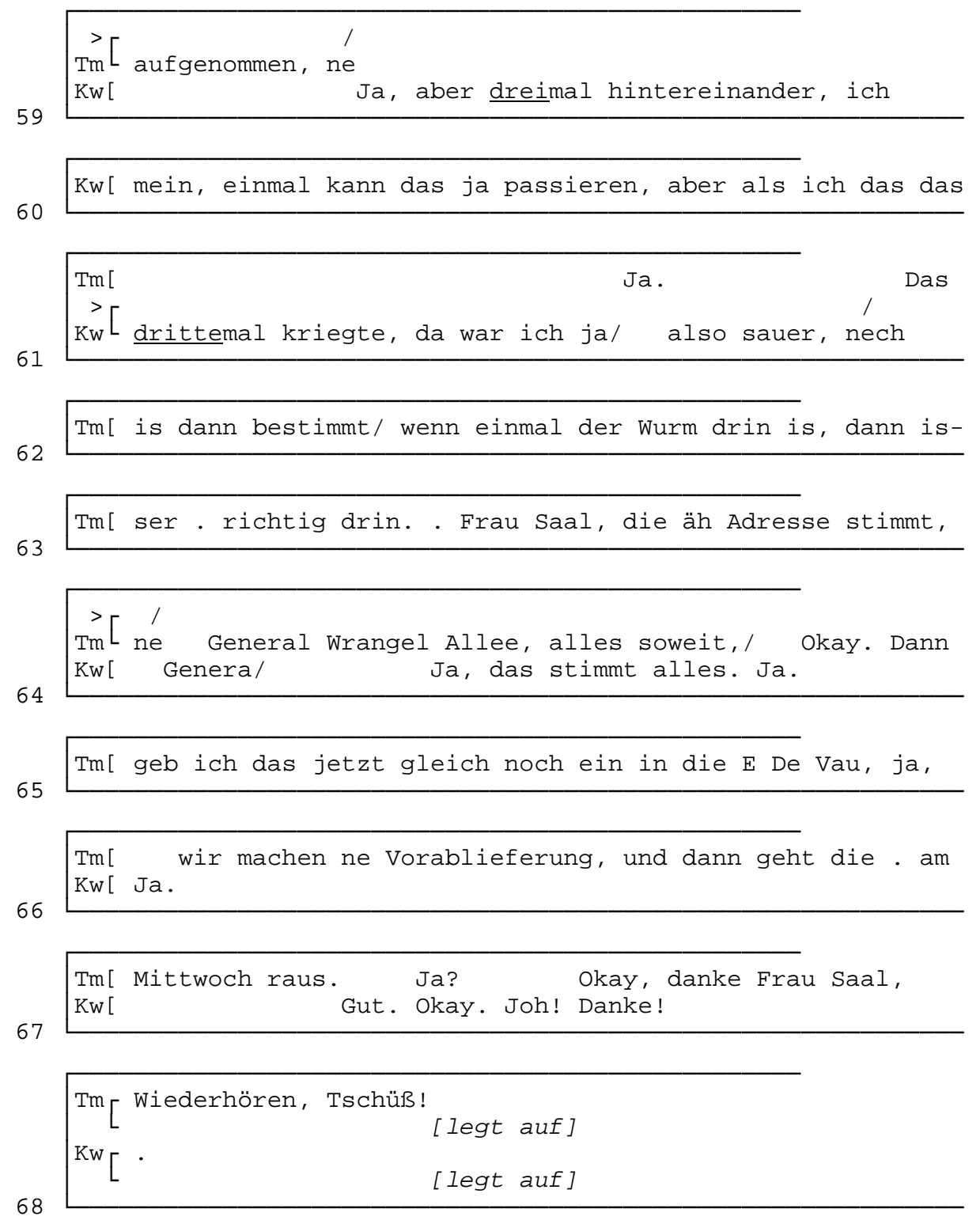

Wie gesagt: Das Gespräch ist insofern erfolgreich, als es zu einer Lösung des Sachproblems führt - die Kundin bekommt, so wird vereinbart, das Produkt so schnell wie möglich zugesandt. Dabei ist deutlich, daß beide, Sachbearbeiter wie Kundin, am Erreichen dieser Lösung mehr interessiert sind als an einer detaillierten Aufarbeitung dessen, was zuvor die Probleme verursacht hat; beide kooperieren also erfolgreich hinsichtlich der Lösung. Trotzdem glückt das Gespräch nur teilweise und einseitig; es weist eindeutig problematische Aspekte auf.

In der folgenden, vorläufigen Analyse werden wir zeigen, inwiefern das vorliegende Gespräch nur in begrenzter Hinsicht glückt und wo es zu Problemen kommt. In Abschnitt 3 wird dann im Rahmen einer Verallgemeinerung geklärt, warum das so ist. Beginnen wollen wir jedoch mit einigen Bemerkungen zu den Voraussetzungen von Reklamationsgesprächen. 


\subsection{Allgemeine Voraussetzungen von Reklamationsgesprächen}

Reklamationen setzen - juristisch gefaßt - das Bestehen eines Kauf-Verkauf-Verhältnisses voraus. Im vorliegenden Beispiel wird zwar bereits diese Voraussetzung vom Reklamationsbearbeiter zwischenzeitlich hinterfragt (FI 19/20) ${ }^{1}$ - die Kundin kann sich jedoch darauf berufen, daß sie zu einem früheren Zeitpunkt bereits ein größeres Produkt erworben hat, zu dem sie nun lediglich Verbrauchsmaterial und Zubehörteile bestellt. Die Möglichkeit solcher Nachbestellungen ist Teil der selbstverständlichen Erwartungen, die sich für die Kundin an die allgemeinen Gepflogenheiten des Geschäftslebens knüpfen.

Neben dem Vorliegen eines Kauf-Verkauf-Verhältnisses setzen berechtigte Reklamationen eine Verletzung der vertraglichen Pflichten seitens des Unternehmens voraus. Auf seiten der Kunden wirkt sich ein entsprechender Reklamationsanlaß als Störung der normalen Abläufe des Alltags aus. Der Bedarf, der bei einem Kauf bzw. einer Bestellung besteht, bleibt zunächst unbefriedigt. Die Kundin in unserem Beispiel etwa kann das früher gekaufte Produkt ohne die bestellten Teile nicht nutzen. Hinzu kommt, daß sie trotzdem die Kaufsumme verfügbar halten muß - schließlich kann die Bestellung jederzeit ausgeliefert werden. Die Kundin sieht also ihre begründeten Erwartungen an den Kauf- und Lieferprozeß enttäuscht. Reklamationsgesprächen geht somit eine gewisse Verärgerung der Kunden über das Unternehmen voraus. Deshalb ist in diesen Gesprächen mit emotionalen Reaktionen und Vorwurfshandlungen der Kundlnnen zu rechnen.

Zugleich besteht bei Kundlnnen die Erwartung, daß die verletzten Ansprüche wieder repariert werden. Damit sie überhaupt den Aufwand einer Reklamation auf sich nehmen, müssen sie dem Unternehmen ein gewisses Maß an Problemlösefähigkeit und -bereitschaft unterstellen - andernfalls würden sie auf ihre Ansprüche verzichten und zur Konkurrenz abwandern oder gleich einen Anwalt einschalten. Viele Unternehmen versuchen, das Vertrauen zu ihnen zu fördern, indem sie Reklamationsabteilungen einrichten und die Reklamationsbearbeitung - teilweise mit beträchtlichem Aufwand (vgl. Hansen/Schönheit 1985, 1987) - professionalisieren. ${ }^{2}$

In Reklamationsgesprächen nun wirken sich die genannten Erwartungen als Interesse und aktive Mitwirkung der Kunden an einer Problembearbeitung aus. Insgesamt kennzeichnen also sowohl Verärgerung als auch Problemlösungsinteresse der Kunden den spannungsgeladenen Rahmen, in dem diese Gespräche stattfinden. In unserem Beispiel wird diese Spannung deutlich, wenn sich in den Beiträgen der Kundin Beschreibungen eigener Emotionen (z.B. Fl. 13, FI. 61) und Ungeduldsäußerungen (FI. 14, Fl. 34-36) mit Phasen abwechseln, in denen sie sich deutlich um den zweckgerichteten, d.h. auf eine Problemlösung abzielenden Fortgang des Diskurses bemüht. Im Sinne der allgemeinen Voraussetzungen von

In Klammern gesetzte Zahlenangaben beziehen sich auf die im Transkript vorgenommene Flächennumerierung.

2 Das Unternehmen, dem unser Beispielgespräch entstammt, hat eine solche Abteilung eingerichtet und dabei großen Aufwand betrieben; einer erfolgreichen und den Kunden zufriedenstellenden Reklamationsbearbeitung wird hier also eine hohe Bedeutung beigemessen. 
Reklamationen ist dieses Verhalten nicht verwunderlich; für Reklamationsgespräche ist es vielmehr typisch.

\subsection{Vorläufige Analyse}

Vor dem Hintergrund dieser allgemeinen Voraussetzungen ist es nun möglich, das Beispielgespräch vorläufig zu analysieren. Es beginnt damit, daß die Kundin, die als Anruferin das Gespräch initiiert, ihr Anliegen vorträgt. Zunächst gibt sie sich als Kundin zu erkennen (sie hat eine Bestellung aufgegeben, FI. 4/5); sie klärt also die institutionelle Beziehung der Beteiligten zueinander. Anschließend skizziert sie die ihrer Ansicht nach wichtigen Aspekte des bisherigen Verlaufs des Kauf-, Liefer- und Reklamationsprozesses (FI. 2-9); dabei benennt sie auch den von ihr reklamierten Sachverhalt (die falsche Ausführung ihrer Bestellung, Fl. 4-6) sowie den aktuellen Anlaß des Anrufs (das Schreiben, in dem die Angabe einer ihr nicht bekannten Auftragsnummer erbeten wird, FI. 10/11). Insgesamt bezieht die Kundin das Gespräch durch zweimaligen Hinweis auf ihre frühere Beschwerde (FI. 3, FI. 7). Die Sequenz endet mit der Aussage der Kundin, daß sie nicht in der Lage ist, auf den letzten Schritt des Unternehmens erwartungsgemäß zu reagieren: Die dem Auftrag zugeordnete Nummer ist ihr nicht bekannt (FI. 10/11).

Der Sachbearbeiter reagiert mit der Mitteilung, daß er sich an den Vorgang erinnern kann (FI. 11/12). Er verzichtet damit zunächst darauf, die von der Kundin begonnene Problemdarstellung inhaltlich zu kommentieren. Dies nimmt die Kundin zum Anlaß, ihre Verärgerung zu äußern und vorwurfsvoll-ungeduldig auf den eigentlichen Grund ihrer Reklamation (die ausbleibenden Waren) zu verweisen; das Schreiben qualifiziert sie als neuerliche Verzögerung. Erst im Anschluß daran versucht der Sachbearbeiter zu beschreiben, was für ihn die weitere Bearbeitung der Bestellung wie der Reklamation blockiert (FI. 15-18): Er kann die Auftragsnummer nicht ermitteln.

Die Auftragsnummer, der Gegenstand sowohl des Schreibens wie dieser Gesprächssequenz, ist tatsächlich für den Fortgang der Reklamationsbearbeitung zentral. Diese Nummern werden jeder Bestellung automatisch zugeordnet; sie dienen der unternehmensinternen, EDV-gestützten Verwaltung. Ohne sie ist die Bestellung, ist der gesamte Vorgang nicht zu finden. Da die Kundin schon mal bei dem Unternehmen gekauft hat, könnte ihr die Existenz einer solchen Auftragsnummer grundsätzlich bekannt sein - was sie wohl nicht weiß, ist, daß diese Nummern ausnahmslos auf jeder Lieferung und jedem Schreiben des Unternehmens an seine Kunden vermerkt werden. Das geschieht, damit die Kunden diese Nummern in Gesprächen als Referenznummern angeben können. Daß die Kundin eine derartige Nummer nicht zu benennen weiß, gilt dem Sachbearbeiter deshalb als Zeichen dafür, daß entgegen ihren Aussagen noch nichts bestellt und geliefert wurde (FI. 19/20): Hätte die Kundin eine Lieferung erhalten, so wüßte sie auch die Nummer.

An dieser Stelle entstehen erste 'Turbulenzen'. Der Sachbearbeiter scheint mit seiner Bemerkung, anders als von der Kundin behauptet, habe sie noch nichts bestellt, ihre einleitende Darstellung als falsch zurückzuweisen. Damit leugnet er zugleich das Bestehen des reklamierten Sachverhalts und folglich die Berechtigung 
der Reklamation. Ist aber die Reklamation nicht berechtigt, so muß auch die von der Kundin geäußerte Verärgerung als unangemessen gelten. Das ist, so gesehen, ein Vorwurf - und die Kundin, die ja wohl nicht weiß, auf Grundlage welcher Schlußfolgerungen der Sachbearbeiter seine Bemerkung macht, reagiert demgemäß irritiert.

So ist ihre sofortige, insistierend vorgetragene Antwort, sie habe doch bestellt (Fl. 20-23), eine deutlich verärgerte Wiederholung dessen, was sie vorher bereits (FI. 2-6) gesagt hat. Auch später, nach Abschluß der Problembearbeitung, hält sie es noch für nötig, auf die Berechtigung ihrer Reklamation hinzuweisen: Sie muß etwas bestellt haben, schließlich hat sie ja auch etwas zugeschickt bekommen (FI. 54-58); zuletzt sucht sie ihre Verärgerung nochmals als angemessen zu rechtfertigen (FI. 59-61).

Diese Entwicklung ist besonders deshalb unglücklich, weil der Sachbearbeiter der Kundin möglicherweise gar nicht auf diese Weise widersprechen wollte: Er nimmt nur eine Bewertung des Sachverhalts gemäß der in der EDV feststellbaren Informationen vor. Dies liegt für inn vor allem deshalb nahe, weil er bei allen seinen Entscheidungen an die in der EDV vorliegenden Informationen gebunden ist. Aus seiner Sicht hat er lediglich die Situation EDV-entsprechend interpretiert und dabei wohl auch eine mögliche Lösung im Auge gehabt. Die abschließenden Partikeln dann eben nämlich kennzeichnen die Bemerkung, es sei noch nichts bestellt, unauffällig als irreal, als kontrafaktische Annahme - etwa im Sinne von Dann tun wir eben so, als wäre nichts bestellt, und ignorieren die Frage der Auftragsnummer. Die handlungspraktische Konsequenz eines solchen Verständnisses der Aussage wäre, daß die alte Auftragsnummer nicht weiter gesucht und die Angelegenheit als neue Bestellung behandelt würde. Und genau das ist es ja auch, was der Sachbearbeiter schließlich als Problemlösung durchführt (FI. 37-51). Die Kundin kann diese Konsequenz natürlich nicht erschließen und reagiert verärgert. Insgesamt scheint es somit so zu sein, als hätte der Versuch des Sachbearbeiters, die Umdefinition des Sachverhalts mit dem Ziel, eine Lösung vorzunehmen, durch eine unglückliche Formulierung zu einer konfliktbeladenen Atmosphäre geführt.

Doch steht die ambivalente Aussage, es sei noch nichts bestellt, nicht allein. Direkt im Anschluß an den Protest der Kundin folgt eine durch Unterbrechungen seitens des Sachbearbeiters (FI. 23, FI. 25, FI. 30) gekennzeichnete Sequenz (FI. 23-30). In dieser drängt er die Kundin, ausführlich zu begründen, warum sie die offensichtlich falschen Pakete hat zurückgehen lassen. Was den Sachbearbeiter hier möglicherweise zu seinen Fragen veranlaßt, ist der Umstand, daß die Firma Sendungen, die sie ohne weitere Bemerkungen zurückerhält, automatisch erneut losschickt. Denn in diesem Fall wird angenommen, daß die Kunden von der Post nicht angetroffen wurden. Die Nichtannahme der Lieferungen führt so lediglich zu erneuten Zustellversuchen. Eine aktive Rückmeldung ist die Voraussetzung dafür, daß das Unternehmen das Problem anders einordnet und behandelt.

Auf die Kundin, die diesen Hintergrund nicht kennt, muß das Verhalten des Sachbearbeiters jedoch ganz anders wirken. Sie sieht erneut ihre Aussagen und die Berechtigung ihrer Reklamation und ihrer Verärgerung in Frage gestellt (vgl. Dann wissen Se doch gar nicht, was da drin gewesen ist (FI. 25/26).

Der Sachbearbeiter wartet nun die vollständige Antwort der Kundin gar nicht ab, unterbricht sie (FI. 30) und beginnt, Vorbereitungen für die Problemlösung zu 
treffen. Er erfragt die Auftragsnummer des Schreibens, mit dem nach der Nummer der ersten Bestellung gefragt wurde (FI. 30-34) - ohne indes dabei zu erklären, was er nun zu tun beabsichtigt. In der Pause, die entsteht, während er in der EDV die Kundendaten aufruft, äußert die Kundin wiederum ihre Verärgerung (FI. 34-37) und formuliert Vorwürfe (FI. 39/40). Abgesehen von dem lapidar-zustimmenden Genau (FI. 37) ignoriert der Sachbearbeiter ihre Beschwerden und führt die Problemlösung durch (FI. 37-51) - nach wie vor, ohne sein Tun zu explizieren. Während dieser Phase stellt er dann fest, daß die Bestellung einen erheblichen sachlichen und preislichen Umfang hat (FI. 42-44).

Mit der Mitteilung des Sachbearbeiters, die bestellten Waren würden Ende der Woche kommen (FI. 50/51), und der Ratifizierung der Lösung durch die Kundin (FI. 48, FI. 51) ist die Bearbeitung des Sachproblems insgesamt vollzogen. Trotzdem insistiert die Kundin nochmal auf der Berechtigung ihrer Reklamation (FI. 51-53, Fl. 54-58, FI. 59-61). Der Sachbearbeiter reagiert darauf zunächst sehr abweisend (FI. 53/54): Zwar ist der von der Kundin gebrauchte Ausdruck Kundendienst nicht ganz korrekt, ihre Aussage ist aber hinreichend verstehbar. Die beiden abschließenden Bemerkungen des Sachbearbeiters (FI. 58/59, Fl. 61-63) zu ihren Beschwerden sind dann viel zu lapidar, als daß sie als Anteilnahme gelten könnten; anstelle des Allgemeinplatzes mit dem Wurm drin hätte der Sachbearbeiter zum Beispiel Erklärungen über die Entstehung der Mißverständnisse abgeben können. Nach dieser Bemerkung leitet er aber direkt zurück zur Frage der Problemlösung, wiederholt die entsprechende Vereinbarung und schließt das Gespräch.

\subsection{Resümee}

Das besprochene Gespräch ist für den Verlauf von Reklamationen in vielen Hinsichten typisch - vor allem, weil es relativ zügig zu einer angemessenen Bearbeitung des Sachproblems führt und weil beide Aktanten sich gleichermaßen um eine Lösung bemühen. So gibt der Sachbearbeiter die Erfordernisse der internen Datenverwaltung auf, verzichtet auf das Feststellen der alten Auftragsnummer und initiiert eine neue Lösung, indem er einen neuen Vorgang anlegt; die Kundin ist regelmäßig bereit, die Äußerung ihres Ärgers zu unterbrechen, um sich an der Bearbeitung zu beteiligen. Das Gespräch ist also insoweit erfolgreich.

Typisch ist indes auch, daß das Gespräch nicht in jeder Hinsicht glückt. Die Äußerungen des Reklamationsbearbeiters, so haben wir gezeigt, können so verstanden werden, daß sie der Kundin Falschaussagen und unangemessenes Verhalten unterstellen (FI. 19/20, Fl. 23/24, FI. 25/26). Deshalb insistiert die Kundin mehrfach vehement auf der Berechtigung ihrer Aussagen und der Angemessenheit ihrer Emotionen (FI. 20-23, FI. 54-58); sie fühlt sich also nicht ernst genommen. Über den Fortgang der Bearbeitung wird sie meistenteils im unklaren gelassen; von einer gemeinsamen Problembearbeitung kann deshalb keine Rede sein. An mehreren Stellen werden ihre vom Sachbearbeiter erst evozierten Rechtfertigungen ignoriert (FI. 30, FI. 37, FI. 53/54), wird sie unterbrochen (FI. 30) und wird das Thema gewechselt (FI. 61-64). Sie muß - besonders bei seinen Ausweichmanövern (FI. 53/54) - insgesamt den Eindruck gewinnen, als sei ihre Reklamation eher eine Belästigung, ein Anspruch, den sie gegen den Widerstand der Firma durchkämpfen 
muß. Die lapidaren Bemerkungen des Sachbearbeiters zur Problemgenese (FI. 58/ 59, Fl. 61-63) endlich tragen nichts zur Aufklärung bei und erwecken den Eindruck, die Serviceprobleme dieser Firma seien eher normal als die Ausnahme. Bedenkt man, daß zu den Zielen, die die Firma mit ihren Serviceabteilungen verfolgt, nicht nur die reibungslose Erledigung von Reklamationen gehört, sondern daß dabei der Kunde auch an die Firma gebunden werden soll, daß also in Reklamationsgesprächen positive Selbstdarstellung, Werbung im persönlichen Kontakt zum Kunden betrieben werden soll, so sind diese Wirkungen durch dieses Gespräch alles andere als sichergestellt.

Nun wären die Ergebnisse dieser vorläufigen Analyse uninteressant, wenn wir annehmen müßten, daß der Sachbearbeiter in diesem Gespräch individuelle Fehler machte oder bösen Willens wäre. Das ist nicht der Fall - wie gesagt: Die Probleme dieses Gesprächs sind typisch. Warum derartige Turbulenzen und Mißverständnisse aber in Reklamationsgesprächen systematisch auftreten, zeigt sich nur, wenn man eine größere Menge von innen betrachtet und entsprechend vom Einzelfall abstrahiert. Wir wollen unter anderem dieser Frage im folgenden Abschnitt nachgehen.

\section{Die diskursanalytische Untersuchung von Reklamationsgesprächen (und ihr Beitrag zum besseren Verständnis der beobachteten Kommunikationspro- bleme)}

Versucht man, sich die Wahrnehmung des analysierten Gesprächs durch die Kundin vorzustellen, so hat sie möglicherweise folgenden Gesamteindruck: Sie ist zuversichtlich, daß ihre Bestellung nun rasch bearbeitet wird. Ihr sachliches Problem nähert sich der Lösung. Sie hat aber hart kämpfen müssen, um sich gegen den ausweichenden und teilweise unfreundlichen Sachbearbeiter durchzusetzen. Wie es zu dem Problem der Fehllieferungen gekommen ist, weiß sie nach wie vor nicht. Ihr Ärger über den Osnabrücker Kundendienst (und möglicherweise über das ganze Unternehmen) besteht ungebrochen fort.

Verglichen mit einer solchen Wahrnehmung, hat die vorläufige Untersuchung des Gesprächs eine Reihe von kommunikativen Problemen genauer identifizieren können. Ganz wesentlich hierfür war, daß das Gespräch in verschriftlichter Form vorlag. Dadurch war es in seinen Einzelheiten genauer nachvollziehbar.

In welchen Punkten geht nun die diskursanalytische Untersuchung von Reklamationsgesprächen über eine alltagsweltliche Wahrnehmung und die vorläufige Untersuchung hinaus?

(1) Die diskursanalytische Untersuchung von Reklamationsgesprächen basiert nicht auf einem einzelnen Gespräch, sondern erfolgt auf der Grundlage eines breiten Korpus authentischer Reklamationsgespräche (vgl. Lalouschek/Menz in Bd. 1).

(2) Die Untersuchung erfolgt auf dem Hintergrund einer expliziten Theorie über Kommunikation und ihre Prinzipien und Regeln. Zentrale Modellvorstellungen dieser diskursanalytischen Auffassung von Gespräch sind u.a. die Konzeption des Gesprächs als einer interaktiven Hervorbringung, die Notwendigkeit einer Perspekti- 
venübernahme für die Verständigung, die Zweckbezogenheit und Musterhaftigkeit von Kommunikation (vgl. Becker-Mrotzek/Meier und Antos in Bd. 1).

(3) Konstitutiv für die Korpusanalyse sind neben diesen theoretischen Grundannahmen eine Reihe von methodischen Analyseprinzipien und -verfahren: so die Sequentialität der Analyse (Äußerung nach Äußerung) und die induktive Entwicklung von Fragestellungen sowie von Beschreibungskategorien aus der Untersuchung der einzelnen Aufnahmen etc.

Auf diese Weise gelangt man zu zwei Ergebnissen: Zum einen lassen sich die Besonderheiten herausarbeiten, die Reklamationsgespräche von anderen Gesprächstypen unterscheiden. Sie führen zu einer Definition, die nicht normativ setzend verfährt, sondern diejenigen Aspekte rekonstruiert, die für die Beteiligten an alltäglichen Reklamationsgesprächen tatsächlich handlungsleitend sind. Zum anderen lassen sich die spezifischen Regeln ermitteln, die für diesen Gesprächstyp konstitutiv sind und nach denen sich Kommunikationsteilnehmer richten. Ein Teil dieser Regeln betrifft die kommunikativen Aufgaben, die ein solches Gespräch ausmachen und die bearbeitet werden müssen, wenn ein solches Gespräch entsprechend seinem Zweck geführt werden soll. Die Gesamtheit dieser Aufgaben bildet das Handlungsschema (die Aufgabenstruktur) von Reklamationsgesprächen.

Sowohl die rekonstruierende Definition wie die ermittelten Regeln - speziell das Handlungsschema - sind Teil einer diskursanalytischen Theorie von Reklamationsgesprächen. Beides erfordert gegenstandsangemessene Kategorien zur Beschreibung. Sie werden induktiv aus der Analyse des Materials unter Berücksichtigung allgemeiner Voraussetzungen und der gesellschaftlichen Zwecke dieser Gespräche entwickelt. Die empirische Untersuchung von Reklamationsgesprächen führt so zu einem genauen und vor allen Dingen zu einem expliziten Verständnis von Reklamationen und erlaubt es, Kommunikationsprobleme in diesen Gesprächen auszumachen. Vor dem gewonnenen theoretischen Hintergrund wird nämlich angebbar, ob die beobachtbaren kommunikativen Aktivitäten bestimmte definierende Bedingungen dieser Gespräche verfehlen bzw. notwendige Aufgaben nicht oder nicht ausreichend bearbeiten.

\subsection{Zur Abgrenzung und Definition von Reklamationsgesprächen}

Dem alltagsweltlichen Verständnis nach gehören Reklamationen in das Spektrum der Aktivitäten, mit denen auf eine Enttäuschung von Erwartungen und/oder eine Beeinträchtigung der eigenen Interessen reagiert wird. Sie haben damit gemeinsame Merkmale mit Beschwerden, Einsprüchen, Widersprüchen, Vorhaltungen, Vorwürfen, (An-)Klagen, Drohungen etc., sie unterscheiden sich aber auch in spezifischer Weise von diesen anderen Formen. Die Methode der Abgrenzung und De-finition von Reklamationen besteht darin, diese Differenzen so weit, wie sie für die an alltäglichen Reklamationsgesprächen Beteiligten handlungsleitend sind, zu rekonstruieren und zu explizieren. ${ }^{3}$

3 Der Prozeß dieser Rekonstruktion und Explikation kann hier nicht im einzelnen vorgeführt werden. Wiedergegeben wird lediglich sein Resultat. 
Ein wesentliches Moment von Reklamationen ist, daß sie sich zwischen Einzelpersonen auf der einen Seite und VertreterInnen einer Institution auf der anderen Seite abspielen. Im Standardfall handelt es sich bei dieser Institution um ein Wirtschaftsunternehmen.

Gegenstand der Reklamation ist ein Produkt oder eine Dienstleistung eines Unternehmens, die ein Kunde gekauft hat. ${ }^{4}$

Anlaß für eine Reklamation ist, daß nach dem Verständnis des Kunden erwartbare oder zugesagte Eigenschaften eines Produkts oder einer Dienstleistung, auf die er aus seiner Sicht einen (vertraglichen) Anspruch hat, wider Erwarten nicht vorliegen. Wesentlich ist also, daß ein Mangel (ein Schaden, eine Beeinträchtigung o.ä.) bezogen auf das Produkt oder die Dienstleistung als sachliches Problem vorliegt und daß nach dem Verständnis des Kunden das Unternehmen für dieses Sachproblem verantwortlich ist. Der Mangel verletzt die Erwartungen und Interessen des Kunden, so daß mit dem Sachproblem auf psychisch-emotionaler Seite eine Erwartungsenttäuschung, Unzufriedenheit, Ärger o.ä. korrespondiert. Die Beeinträchtigung der Interessen ist für den Käufer zudem groß genug, um ihn zu entsprechenden Handlungen - der Reklamation - zu veranlassen. ${ }^{5}$

Ziel der Reklamation ist die Behebung des Mangels und/oder das Rückgängigmachen des Kaufs und/oder (u.U. auch als Kompensation für die psychischemotionale Belastung) eine entsprechende Entschädigung/Wiedergutmachung.

Realisiert werden Reklamationen entweder in einem Reklamationsgespräch oder in schriftlicher Form. In diesem Beitrag behandeln wir nur Reklamationsgespräche. Die Gesprächskonstellation ist dabei im Regelfall die, daß der betroffene Kunde mit einem Vertreter des Unternehmens spricht, der im Standardfall nicht der Urheber des Anlasses der Reklamation ist, der aber gleichwohl die Interessen der Institution zu verteten und zu wahren hat. Üblicherweise ist die Reklamation mit dem Ende des Reklamationsgesprächs nicht erledigt, sondern die Behebung des Mangels findet außerhalb seiner Grenzen statt. Reklamationen setzen sich so häufig aus mehreren (Teil-)Gesprächen zusammen.

Reklamationsgespräche sind ein Spezialfall von Problemlösung. Kunden haben ein Sachproblem, und sie erwarten vom Unternehmen hierfür eine Lösung. Charakteristisch für den Problemlösungsprozeß in Reklamationsgesprächen ist ein dreifaches Spezifikum: Zum einen handelt es sich nicht um eine individuelle, sondern um eine kollektive, partiell auch kooperative Problemlösung. Zum zweiten handelt es sich nicht um ein externes Problem, sondern es wird (zumindest von einer Partei) angenommen, daß eine der beteiligten Parteien Urheberin des Problems ist. Dies nun ist die Grundkonstellation (interpersonaler) Konflikte. Zum dritten ist im

4 Gegenstand von Ein-/Widersprüchen sind - um einige Differenzen zu anderen Interaktionsformen deutlich zu machen - vor allem Entscheidungen einer Institution (in Form von Bescheiden). Gegenstand von Beschwerden sind im Regelfall Handlungen (von Einzelpersonen oder Institutionenvertretern) bzw. deren Resultate. Insofern Dienstleistungen natürlich auch Handlungen sind, überlappen sich Reklamationen und Beschwerden. Charakteristischerweise spricht die Kundin im untersuchten Gespräch auch davon, daß sie sich "beschwert" hat (Fläche 3 und 7).

5 Die Beeinträchtigung von Interessen durch einen Mangel führt längst nicht in allen Fällen zu einer Reklamation. Die empirische Untersuchung von Bruhn (1982, 54) ergibt z.B. bei Fernsehgeräten einen Anteil von 51,1\% und bei Autoreparaturen einen Anteil von 23,4\% von 'unvoiced complaints'. 
Regelfall der direkte Interaktionspartner nicht persönlich der Urheber des Problems, sondern er agiert lediglich im Rahmen der Institution.

Mit dieser Struktur sind im Reklamationsgespräch die beiden Pole von sachlicher, gemeinsamer Problemlösung auf der einen Seite und antagonistischer, persönlicher Konfliktaustragung auf der anderen Seite angelegt. Je nach Definition durch die Beteiligten kann das Reklamationsgespräch in Richtung auf einen dieser Pole entwickelt werden, es kann aber auch im Rahmen von Interaktionsdynamik zwischen den Polen hin und her schwanken oder sich von einem Pol zum anderen entwickeln. Diese Struktur macht Reklamationsgespräche zu einem ebenso interessanten und vielfältigen wie problematischen Interaktionstyp mit sehr variablen Entwicklungsmöglichkeiten.

\subsection{Das Handlungsschema von Reklamationen}

Die Diskursanalyse versteht Gespräche als eine komplexe Hierarchie von Aufgaben, die von den Kommunikationspartnern gemeinsam zu bewältigen sind. Für jeden Gesprächstyp läßt sich - gestützt auf umfangreiche empirische Korpusanalysen eine spezifische Aufgabenstruktur herausdestillieren. Diese hierarchische Aufgabenstruktur wird als Handlungs- oder Interaktionsschema bezeichnet. ${ }^{6}$ Das Handlungsschema rekonstruiert Erwartungen sowohl der Gesprächsbeteiligten wie auch der anderen Mitglieder der betreffenden Kultur über notwendige Aufgaben (und ihre prinzipielle/sachlogische Abfolge), die zur Realisierung des Zwecks des jeweiligen Gesprächstyps interaktiv bearbeitet werden müssen, sowie über mögliche kommunikative Aktivitäten, mit denen diese Aufgaben realisiert werden können.

Die Aufgaben sind unterschiedlich allgemein und können hierarchisch voneinander abhängen. Manche Aufgaben sind Aufgaben von Interaktion generell (Organisation des Rederechts, Organisation von Themenwechseln oder Wechseln des Interaktionstyps), andere sind spezifisch für einen bestimmten Interaktions- oder Gesprächstyp. Das Handlungsschema benennt hauptsächlich solche gesprächstypspezifischen Aufgaben. An der Lösung der Aufgaben sind die Gesprächsteilnehmer grundsätzlich gemeinsam beteiligt. Der Beitrag zu ihrer Lösung kann aber unterschiedlich auf die Interaktionspartner verteilt sein. Ebenso können einzelne $\mathrm{Ge}-$ sprächspartner vorrangig für die Lösung bestimmter Aufgaben zuständig sein.

In konkreten Gesprächen müssen - je nach den Gegebenheiten des Einzelfalls nicht alle Aufgaben des Handlungsschemas und auch nicht in der angegebenen Reihenfolge bearbeitet werden. Einzelne Aufgaben des Schemas können in mehreren 'Anläufen' oder Runden an verschiedenen Stellen des Gesprächs bearbeitet werden.

Die Herausarbeitung eines Handlungsschemas ist ein empirischer Prozeß. In der Folge der Analyse von einzelnen Gesprächen, die vorverständlich zu einem Kommunikationstyp gehören, wird auf der Grundlage des jeweiligen Vorwissens eine Hypothese über die Aufgabenstruktur des Gesprächstyps entwickelt. Auf ihrer

6 Zum Begriff des Handlungsschemas und zu einer Darstellung des Handlungsschemas für Beratungsgespräche vgl. Kallmeyer (1985). 
Basis werden weitere Gespräche analysiert und das Handlungsschema ggf. entsprechend modifiziert. Nach aller Erfahrung wird die Notwendigkeit von Modifikationen mit der Zahl der Analysen geringer. Entwicklung und Modifizierung des Handlungsschemas als eines theoretischen Konstrukts und empirische Analyse sind interdependent. Das Handlungsschema ist also in keiner Weise universell oder letztgültig, sondern reflektiert den jeweiligen Stand der empirischen Analyse und das analysierte Material (in seiner Spezifik). D.h. auch, daß ein Handlungsschema adaptiert werden muß und kann, sofern spezifische Materialien - z.B. ein bestimmter Typ von Reklamationsgesprächen - analysiert wird.

Wir möchten im folgenden im Anschluß an Fiehler und Kindt (1994) ein Handlungsschema für Reklamationsgespräche vorstellen, wie es sich aus dem gegenwärtigen Stand unserer Analysen ergibt. Wir werden dabei die Aufgabenhierarchie dieses Gesprächstyps entwickeln, indem wir von den allgemeineren zu den konkreteren Aufgaben (im Schema also von links nach rechts) voranschreiten und die Aufgaben jeder Hierarchieebene zusammen erläutern.

\section{Handlungsschema / Aufgabenstruktur von Reklamationsgesprächen}

Gesprächseröffnung

- Begrüßung

- Identifizierung

- Anknüpfung an vorausgegangene Gesprächskontakte/Interaktionen

- Vorgreifende Verdeutlichung des folgenden Kommunikationstyps

- Ouvertüre

\section{Emotions- und Beziehungsbearbeitung (frei plazierbar)}

\section{- Emotionsbearbeitung}

Manifestation von Emotionalität (Enttäuschung, Ärger etc.) Vorwürfe

Reaktion auf Emotionalität

Anteilnahme

Vorwurfsbearbeitung

- Beziehungskonstitution

Imagepflege beider Seiten

Beziehungspflege

\section{Bearbeitung des Sachproblems}

- Problemexplikation und Voraussetzungsklärung

Klärung der personellen Zuständigkeit

Darstellung des Problems

Problemformulierung

Genese des Problems (z.B. Erzählung/Bericht)

Problembewertung

Problemklärung 


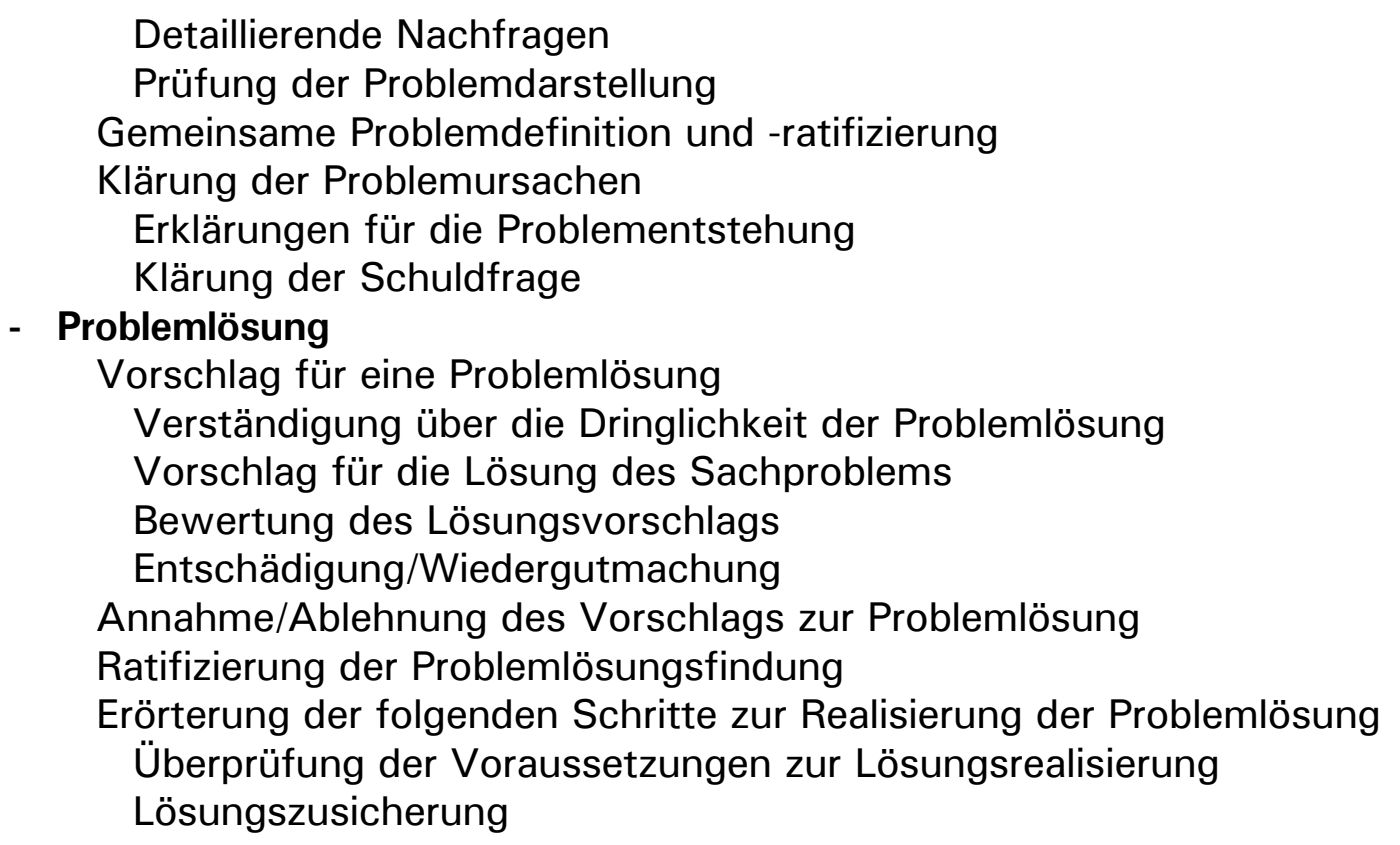

Weitere Durchläufe durch das Handlungsschema

Gesprächsbeendigung

- Rekapitulation und Ratifizierung des weiteren Procedere

- Danksagung

- Verabschiedung

Abb.1: Handlungsschema / Aufgabenstruktur von Reklamationsgesprächen

Zu Beginn von Reklamationsgesprächen sind die üblichen interaktionseröffnenden Aufgaben zu bearbeiten: Begrüßung ${ }^{7}$, Identifizierung und ggf. die Anknüpfung an vorausgegangene Gesprächskontakte sowie eine vorgreifende Verdeutlichung des folgenden Aktivitätstyps. Auf sie soll nicht näher eingegangen werden. In die eröffnenden Aufgaben können aber auch Elemente einer (fakultativen) 'Ouvertüre' eingelagert sein. Ouvertüren sind dem eigentlichen Reklamationsgespräch vorgelagerte Aktivitäten, in denen die reklamierende Person anhand einer anderen Thematik ihre Enttäuschung bzw. Verärgerung stellvertretend und vorgreifend zum Ausdruck bringt (z.B. Probleme, die zuständige Stelle ausfindig zu machen; Probleme der Erreichbarkeit des Gesprächspartners o.ä. (vgl. das Transkriptionsbeispiel bei Antos 1989).

Auf der allgemeinsten Ebene lassen sich im Handlungsschema von Reklamationsgesprächen zunächst Aufgaben der Emotions- und Beziehungsbearbeitung und der Bearbeitung des sachlichen Problems unterscheiden. Wie gesagt darf man sich diese nicht als getrennt und nacheinander zu bearbeitende Aufgaben vorstellen. Das Handlungsschema ist generell nicht im Sinne einer linearen, die oberflächliche Abfolge wiedergebenden Auflistung zu lesen. So kann etwa mit einer einzelnen

7 Die im folgenden kursiv gesetzten Ausdrücke sind Aufgaben des Handlungsschemas. 
Äußerung sowohl zur Emotionsbearbeitung wie auch zur Bearbeitung des sachlichen Problems beigetragen werden.

$\mathrm{Zu}$ den Aufgaben der Emotionsbearbeitung gehören zum einen die Manifestation von Emotionalität durch den Kunden, die durch die Nichterfüllung seiner Erwartungen hinsichtlich des Produkts/der Dienstleistung loder durch den bisherigen Gang der Reklamation) hervorgerufen worden ist, vor allem aber die Reaktion auf Emotionen des Kundens. Die Emotionalität des Kunden kann sich in vielerlei Formen manifestieren: in Vorwürfen, in einer entsprechenden Aufgeregtheit oder Aufgebrachtheit bei der Darstellung des Problems, in einer 'verbohrten' Argumentation etc. (vgl. Fiehler 1990). Eine Reaktion auf die Emotionalität erfordert die Manifestation von Anteilnahme (zum Anteilnahmemuster vgl. Fiehler 1990) und die Bearbeitung der Vorwürfe.

Neben der Bearbeitung der Emotionen des Kunden stehen Aufgaben der Beziehungskonstitution. Beide Seiten haben Aufgaben der Imagepflege zu bearbeiten. Für den Kunden geht es z.B. darum, sich als 'kompetenten' Reklamierenden zu präsentieren und den Verdacht des Querulantentums oder der Schadensreiterei nicht aufkommen zu lassen. Dem Unternehmen wird daran gelegen sein, sich als sachlich, kompetent und entgegenkommend darzustellen. Nach abgewickelter Reklamation geht es häufig darum, das Geschehen in seinen Auswirkungen auf die Beziehung zwischen Kunde und Unternehmen zu thematisieren (Beziehungspflege).

Neben der Emotions- und Beziehungsbearbeitung stehen die Aufgaben der $\mathrm{Be}$ arbeitung des Sachproblems. Sie gliedern sich in die großen Komplexe der Problemexplikation und Voraussetzungsklärung sowie der Problemlösung.

Im Rahmen der Problemexplikation und Voraussetzungsklärung sind fünf Teilaufgaben zu unterscheiden: Es muß geklärt werden - häufig gleichzeitig mit der Darstellung des Problems -, ob der Gesprächspartner in sachlicher Hinsicht und in Hinsicht auf die Entscheidungskompetenz für Lösungen die zuständige Instanz ist (Klärung der personellen Zuständigkeit). Überwiegend eine Aktivität des Kunden ist dann die Darstellung des Problems. Sie kann unterbrochen oder unterstützt werden durch die Problemklärung, die weitgehend in den Zuständigkeitsbereich des Unternehmensvertreters fällt. Beides sind Voraussetzungen, um zu einer Festlegung des gemeinsam zu bearbeitenden Problems zu kommen /Gemeinsame Problemdefinition und -ratifizierung). Die fünfte Aufgabe ist die Klärung der Problemursachen.

Die Darstellung des Problems umfaßt als Teilaufgaben eine Problemformulierung, die als Problembenennung oder -beschreibung, als Benennung unerfüllter Erwartungen oder als Beschwerde vorgebracht werden kann, einen Bericht oder eine Erzählung über die Genese des Problems, wobei der Aspekt der Problemerkenntnis und mögliche Problemursachen eine Rolle spielen können, sowie eine Problembewertung, die die Bedeutsamkeit des Problems thematisiert und (negative) Konsequenzen des Problems benennt.

Wesentliche Aktivitäten der Problemklärung durch den Unternehmensvertreter sind detaillierende Nachfragen, die zur Klärung der Problemsicht beitragen, sowie die Prüfung der Problemdarstellung, was das Sachkundigmachen durch Einholen von Informationen oder eine Sachprüfung umfaßt. Eine mögliche Konsequenz dieses Punktes ist, daß das Reklamationsgespräch unterbrochen oder vertagt wird. 
Dies verweist auf den generellen Punkt, daß Reklamationen häufig nicht in einem Zug durchgeführt werden. ${ }^{8}$

Die wichtige nächste Aufgabe besteht darin, konsensuell eine Problemauffassung festzulegen und zu ratifizieren, was gemeinsam bearbeitet und gelöst werden soll (gemeinsame Problemdefinition und -ratifizierung). Dieses Problem kann von dem abweichen, was der Reklamierende zunächst dargestellt hat.

Die zwei Teilaufgaben im Rahmen der Klärung der Problemursachen sind Erklärungen für die Problementstehung und die Klärung der Schuldfrage. Bei den Erklärungen kann es sich um eine sachliche Aufklärung über die Problementstehung handeln oder um eine Konstatierung von Unerklärbarkeit bzw. höherer Gewalt. Die Klärung der Schuldfrage, also der Verantwortlichkeit, umfaßt die Schuldzuweisung und die Schuldanerkenntnis bzw. -abweisung. Im Fall einer Schuldanerkenntnis, die allerdings im Regelfall nicht explizit erfolgt, können Aktivitäten der Entschuldigung folgen. Wird die Schuld abgewiesen, muß erneut in die Klärung der Verantwortlichkeit eingestiegen werden.

Die Problemlösung setzt sich aus vier Teilaufgaben zusammen. Es muß ein Vorschlag für eine Problemlösung gemacht werden, worauf sich die Annahme oder Ablehnung des Vorschlags anschließt. Im Falle der Ablehnung müssen weitere Lösungsvorschläge gemacht werden. Wird der Vorschlag angenommen, folgt die gemeinsame Ratifizierung der Lösungsfindung. Da die Lösung in den meisten Fällen nicht unmittelbar zu realisieren ist, folgt die Erörterung der folgenden Schritte zur Realisierung der Problemlösung. Häufige Teilaktivitäten in diesem Rahmen sind die Überprüfung, ob alle Voraussetzungen zur Realisierung vorliegen, und die dezidierte Zusicherung der Realisierung der gefundenen Lösung.

Dem Vorschlag für eine Problemlösung geht häufig eine Verständigung über die Dringlichkeit der Problemlösung voraus. Der Vorschlag für die Problemlösung umfaßt den Vorschlag für die Lösung des Sachproblems, die Bewertung des Lösungsvorschlags und eine Entschädigung oder Wiedergutmachung für die entstandenen Beeinträchtigungen und Unannehmlichkeiten.

An einen ersten Durchgang durch das Handlungsschema können sich weitere häufig verkürzte - Durchläufe anschließen. Dies stimmt mit der generellen Rundenstruktur von Interaktion überein. In diesen weiteren Durchläufen werden z.B. Aufgaben behandelt, die bisher nicht bearbeitet wurden, es kann eine erweiterte Behandlung schon bearbeiteter Aufgaben stattfinden, und die Aufgabenbearbeitung kann generell unter dem Aspekt ihrer Auswirkungen auf Stand und Entwicklung der wechselseitigen Beziehung thematisiert werden.

Abschließend erfolgen Beendigungsaktivitäten: die Rekapitulation und Ratifizierung des weiteren Procedere, Danksagungen und die Verabschiedung.

8 Vgl. Bruhn $(1982,56)$ zur Häufigkeit von Beschwerdekontakten im Rahmen einzelner Reklamationen. Bei Fernsehgeräten werden 54\% der Reklamationen, bei Autoreparaturen nur 17,4\% in einem Zug abgewickelt. Die Untersuchung erfaßt bis zu sechs Kontakte (4,4\% bzw. 17,4\%). 
3.3 Die Leistungsfähigkeit von Definition und Handlungsschema für die Analyse von Kommunikationsproblemen im untersuchten Reklamationsgespräch

Betrachten wir nun das in Abschnitt 2 vorgestellte Gespräch vor dem Hintergrund des Handlungsschemas, so läßt sich genau angeben, was in dem Gespräch passiert (welche Aufgaben bearbeitet werden) - und eben auch, was nicht geschieht. Für jede Äußerung muß geprüft werden, welche Aufgaben mit ihr bearbeitet werden. Diese Analyse kann hier nicht im Detail vorgeführt, aber es sollen ihre Ergebnisse vorgestellt werden.

Bei der Bearbeitung des Sachproblems bewegt sich der Reklamationsbearbeiter nur im Bereich der Problemklärung. Die Klärung der personellen Zuständigkeit ist in diesem Gespräch nicht notwendig. Die Darstellung des Problems wird weitgehend von der Kundin getragen. Ganz wesentlich ist, daß eine gemeinsame Problemdefinition und -ratifizierung nicht stattfindet. Ferner unterbleibt eine Klärung der Problemursachen. Weder werden Erklärungen für die Problementstehung gegeben, noch erfolgt eine Klärung der Schuldfrage. Weitgehend bewegt sich der Reklamationsbearbeiter in der Aufgabe der Problemlösung (spätestens ab FI. 37), wobei auch eine gemeinsame Ratifizierung der Problemlösungsfindung nicht erfolgt.

Aufgaben der Emotions- und Beziehungsbearbeitung bearbeitet der Sachbearbeiter nicht. Er zeigt keine angemessene Reaktion auf die Emotionalität der Kundin, ein folgenschwerer Fehler, wie aus anderen Untersuchungen zu Reklamationsgesprächen und aus Arbeiten zur Konfliktkommunikation bekannt ist. Er betreibt auch keine Image- und Beziehungspflege. D.h. der Sachbearbeiter beschränkt sich ausschließlich auf die Bearbeitung des Sachproblems, und diese erfolgt stark reduziert.

Die Kundin hingegen bearbeitet alle Aufgaben, für die sie hauptsächlich zuständig ist, insbesondere leistet sie eine genaue Darstellung des Problems, wobei sie mehrfach die Klärung der Problemursachen anmahnt. Sie bleibt damit aber erfolglos. Ihre Manifestationen von Emotionalität sind zahlreich, es erfolgt aber - wie gesagt keine Reaktion darauf. Die Emotionalität bricht deshalb immer wieder von neuem ins Gespräch, und in verstärktem Maße insbesondere dann, als das Sachproblem gelöst erscheint (vgl. Fl. 51-53 und 59-61).

Struktur und Besonderheiten des konkreten Einzelgesprächs werden so vor der Folie des Handlungschemas transparent. Von seiten des Reklamationsbearbeiters ist eine spezifisch reduzierte Realisierung des Schemas erkennbar. Die Defizienz der Schemabearbeitung hat für die Beteiligten, insbesondere für die Kundin, eine wesentliche Konsequenz. Was passiert, muß ihr als problematische Kommunikation erscheinen. Ihre Erwartungen darüber, was ein Reklamationsgespräch leisten soll, werden nicht erfüllt.

Im Rahmen des Gesprächs lassen sich drei zentrale Probleme identifizieren: (1) die fehlende Reaktion auf Emotionalität, (2) eine mangelnde Perspektivenübernahme und (3) die unzureichende Explikation unterschiedlicher Wissenshintergründe und Relevanzen. Das erste Problem braucht nicht mehr kommentiert zu werden. Auch beim zweiten Problem sind die Beteiligungsrollen unterschiedlich: Die Kundin übernimmt - wie ihre Kooperativität belegt - weitgehend die Perspektive des Sachbearbeiters, dies gilt aber nicht für ihn. Er versetzt sich nicht in die Lage 
der Kundin und versucht nicht zu verstehen, worum es ihr - außer der Lösung des Sachproblems - noch gehen könnte. Er verfehlt so z.B. ihr Bedürfnis nach Aufklärung des Sachverhalts. Letztlich expliziert der Reklamationsbearbeiter zu wenig sein Wissen über organisatorische Abläufe im Unternehmen, was der Kundin zu einem besseren Verständnis der Situation verhelfen könnte. Der Sachbearbeiter macht sich nicht klar, daß dieses Wissen zwar für ihn, nicht aber für die Kundin selbstverständlich ist.

Alle drei Probleme, die sich aus spezifischen Reduktionen des Handlungsschemas ergeben, lassen sich wahrscheinlich auf eine gemeinsame Ursache - professionelle Routine - zurückführen. Eine gemessen an den eigenen Interessen möglichst effiziente Bearbeitung des 'Falls' gewinnt dabei die Oberhand über eine Orientierung an den individuellen Interessen der Kundin. Sie werden zunehmend nicht mehr wahrgenommen und bearbeitet. Der Professionelle meint vorab immer schon zu wissen, was der Kunde wünscht, ohne im Einzelfall diese Unterstellungen auch zu verifizieren. Die Herausarbeitung der Individualität des Anliegens, die Explikation des eigenen Hintergrundwissens und die Bearbeitung von Emotionalität erscheinen dagegen als Zeit beanspruchende Störfaktoren. In Wirklichkeit erweisen sich aber solche 'Abkürzungen' als insgesamt zeitaufwendiger, weil die nicht bearbeiteten Aufgaben an den verschiedensten Stellen des Gesprächsgeschehens immer wieder eingefordert werden und sie so dem Gespräch eine zeitraubende Schleifenstruktur aufprägen. ${ }^{9}$

Neben den genannten läßt sich eine Reihe weiterer typischer Kommunikationsprobleme in Reklamationsgesprächen feststellen. Im folgenden Abschnitt sollen sie systematisch behandelt werden.

\section{Häufige Probleme in Reklamationsgesprächen und ihre Lösung}

Wenn ein Unternehmen die Effizienz von Reklamationsgesprächen verbessern will, ist es wichtig, daß die mit Reklamationen befaßten MitarbeiterInnen auf das Auftreten und die Lösung relevanter Problemtypen vorbereitet sind. Definition und Handlungsschema aus Abschnitt 3 liefern hierfür eine wesentliche theoretische Grundlage, weil sie präzise Aussagen darüber ermöglichen, an welcher Stelle eines Reklamationsgesprächs welche Probleme auftreten können. Ein Problem liegt nämlich immer dann vor, wenn eine notwendige Aufgabe des Handlungsschemas nicht (ausreichend) erfüllt wurde. Somit ist es zweckmäßig, wenn Reklamationsbearbeiterlnnen in Kommunikationsschulungen zunächst bewußt gemacht wird, welche kommunikativen Aufgaben sie und ihre Kunden an welcher Stelle des Gesprächsverlaufs gemeinsam zu bewältigen haben. Hätte der Mitarbeiter im Beispielgespräch aus Abschnitt 2 ein expliziteres Wissen über das Aufgabenprofil von Reklamationsgesprächen gehabt, dann wäre ihm möglicherweise klar gewesen, daß er Emotionen in den Äußerungen von Kunden nicht ignorieren sollte und daß er die Kunden genauer über seine Vorgehensweise bei der Problembearbeitung informieren muß, um Mißverständnisse zu vermeiden.

9 Im vorliegenden Gespräch sieht man dies deutlich an den Reaktionen der Kundin in den Flächen 34-37 und 51-61. 
Für eine erfolgreiche Lösung von Kommunikationsproblemen reicht es allerdings nicht aus, wenn man die Probleme benennen kann und weiß, wo sie üblicherweise auftreten. Vielmehr ist es auch notwendig, mögliche Ursachen dieser Probleme, also Gründe für die Nichterfüllung von Aufgaben des Handlungsschemas, zu kennen und ein explizites Wissen über effiziente Lösungsverfahren zu besitzen. Zu beiden Aspekten können hier Ergebnisse der Diskursforschung präsentiert werden. Betrachten wir zunächst die Ursachenfrage, so ist wichtig, daß oft nicht individuell, sondern strukturell bedingte Faktoren Auslöser für die Problementstehung sind. Nach den vorliegenden Untersuchungen über Reklamationsgespräche sind für die besonders gravierenden und häufig auftretenden Kommunikationsprobleme (vgl. Fiehler/Kindt 1994; Schnieders im Druck) hauptsächlich folgende drei Typen von Ursachen verantwortlich.

(1) Kunden und Reklamationsbearbeiterlnnen kategorisieren oder bewerten das zugrundeliegende Sachproblem verschieden.

(2) Die Gesprächspartner haben unterschiedliche Vorstellungen über die Vorgehensweise bei der Problembearbeitung.

(3) Wissens- und Interessendifferenzen der Beteiligten hinsichtlich bestimmter Sachverhalte führen zu Verständigungsproblemen oder Konflikten.

Entsprechende Problemursachen liegen auch dem Gespräch aus Abschnitt 2 zugrunde. Eine Differenz in der Problembewertung zeigt beispielsweise die topische, problemzudeckende Formulierung wenn einmal der Wurm drin is, dann isser . richtig drin, mit der der Mitarbeiter auf die Äußerung der Kundin ich mein, einmal kann das ja passieren, aber als ich das das dritte Mal kriegte, da war ich ja/ also sauer, nech reagiert. In dieser Reaktion stuft der Mitarbeiter die Relevanz des Sachproblems und die Verantwortlichkeit des Unternehmens durch die Behauptung einer quasi naturgesetzlichen Zwangsläufigkeit zurück. Daß bei Mitarbeiter und Kundin unterschiedliche Vorstellungen über die Problembehandlung bestehen, haben wir schon für die Aufgabe, auf Kundenemotionen einzugehen, gezeigt. Und schließlich wurden in Abschnitt 2 auch die negativen Auswirkungen bestimmter Wissensdifferenzen demonstriert: An mehreren Stellen im Gespräch macht der Mitarbeiter Äußerungen, welche die Kundin deshalb nicht im intendierten Sinne verstehen kann, weil ihr bestimmte Kenntnisse über Bearbeitungsvorgänge im Unternehmen fehlen.

Kommunikationsprobleme, die durch die drei genannten Arten von Ursachen ausgelöst werden, kommen nicht nur in Reklamationsgesprächen, sondern auch in vielen anderen Interaktionstypen vor. Deshalb kann man für die Problemlösung auf generelle Verständigungssicherungsstrategien zurückgreifen, wie sie seit längerer Zeit in der Diskursforschung formuliert werden. Zentrale Lösungsverfahren bei Vorliegen von Differenzen sind

- die Explikation von Voraussetzungen,

- die wechselseitige Perspektivenübernahme und

- die Entwicklung gemeinsamer Zielsetzungen. 
Was diese Verfahren konkret bedeuten, wird bei den nachfolgenden Beispieldiskussionen deutlich werden. Dabei wollen wir jeweils eine Äußerung oder Gesprächspassage zitieren, an der sich prototypisch ein bestimmtes Kommunikationsproblem zeigt. Anschließend werden mögliche positive oder negative Reaktionsweisen erläutert und teilweise anhand von Daten aus unserem Korpus veranschaulicht. Die im einzelnen zu diskutierenden Probleme sollen entsprechend der Typenunterteilung (1) - (3) in den Unterabschnitten 4.1 bis 4.3 dargestellt werden.

\subsection{Kommunikationsprobleme aufgrund von Beurteilungsdifferenzen bei der Ex- plikation des Sachproblems}

Grundsätzlich können Einschätzungsunterschiede hinsichtlich jeden Sachverhalts, der in einem Reklamationsgespräch verhandelt wird, zu Verständigungsschwierigkeiten zwischen den Gesprächspartnern führen. Besonders problematisch für ein Reklamationsgespräch ist es aber, wenn schon bei der Problemexplikation Beurteilungsdifferenzen auftreten. Diesbezüglich erweisen sich insbesondere sieben Arten von Problemen (4.1.1 - 4.1.7) als gravierend.

\subsubsection{Problemanzweiflung}

Für bestimmte Aspekte der Darstellung und Definition des Sachproblems liegt die primäre Beurteilungskompetenz bei den Kunden. Deshalb werden Reklamationsgespräche immer dann konfliktträchtig, wenn Reklamationsbearbeiterlnnen zugehörige Kundendarstellungen direkt oder indirekt anzweifeln und somit - gewollt oder ungewollt - die Glaubwürdigkeit oder Kompetenz des Kunden in Frage stellen. Dies gilt insbesondere für den Fall, daß Sachverhalte bestritten werden, deren Geltung durch die unmittelbare Wahrnehmung oder Erfahrung von Kunden begründet ist. Ein prototypisches Beispiel hierfür stammt aus den Daten von Antos (1989), bei dem eine Reklamationsbearbeiterin den vom Kunden reklamierten Mangel für unmöglich hält: denn es kann nicht sein, daß wir Ihnen ein Gerät liefern, ein komplettes Gerät liefern, wo das eine Teil aus diesem Jahr ist und das andere aus $n$ paar Jahren davor. Das gibts nich. Selbst wenn berechtigte Zweifel an der Problemdarstellung oder Entstehungserklärung von Kunden bestehen, ist es in jedem Fall zweckmäßig, vorsichtiger zu formulieren. Zudem muß man immer damit rechnen, daß in der Praxis sein kann, was nach der Theorie eigentlich nicht sein darf.

\subsubsection{Differierende Erwartungen}

Eine andere Art von Differenzen, auf deren negative Konsequenzen Reklamationsbearbeiterlnnen vorbereitet sein müssen, besteht darin, daß mit dem Reklamationsgegenstand häufig unterschiedliche Erwartungen verbunden sind (Erwartungskoordinationsproblem; vgl. Kindt/Weingarten 1984). Eine Erwartungsdifferenz äußert 
sich z.B. im folgenden Dialog, in dem ein Kunde moniert, daß inm die Angestellte eines Reisebüros nicht - wie möglich - eine verbilligte Fahrkarte ausgestellt hat.

\section{K: Warum haben Sie das nicht gemacht? \\ M: Dann hätten Sie mir sagen können, ob Sie demnächst 26 wer- den. Also es kann durchaus sein, es gibt diese Twen-Tours- Fahrkarten. Das ist richtig. Aber ich kann nicht jeden Kun- den fragen, wie alt ist er, ne.}

Die Mitarbeiterin weist hier die Erwartung des Kunden zurück, daß sie in jedem Fall unaufgefordert die Voraussetzungen für das Ausstellen einer verbilligten Fahrkarte prüfen müsse. In einer solchen Situation läßt sich die Entstehung eines Konflikts nur verhindern, wenn man die Berechtigung der differierenden Erwartungen aus der jeweiligen Teilnehmerperspektive argumentativ klärt und das Ergebnis in der Problemdefinition berücksichtigt. Genau dies wird häufig nicht getan, und statt dessen insistieren die Beteiligten auf der ausschließlichen Geltung ihrer eigenen Position. Daß nicht auf ihre Erwartungen eingegangen wird, verärgert Kunden insbesondere dann, wenn sie 'alte Kunden' sind oder 'in gutem Glauben' bestimmte Vorleistungen für die Abwicklung des Geschäfts erbracht haben.

\subsubsection{Unterschiedliche Normalitätsvorstellungen}

Ein anderer Problemtyp liegt vor, wenn die Erwartungen an den Reklamationsgegenstand zwar identisch sind, ihre Nichterfüllung aber von Kunde und Reklamationsbearbeiterln unterschiedlich bewertet wird. Wie gravierend eine Erwartungsabweichung ist, hängt einerseits von der Art des Sachproblems und andererseits vom Grad der Verantwortlichkeit für sein Auftreten ab. In beiden Aspekten sind die Vorstellungen von Kunde und Unternehmen darüber, ob Abweichungen unter bestimmten Randbedingungen als normal bzw. tolerierbar gelten können, sehr unterschiedlich. Beispielsweise wird zwar die Nichteinhaltung eines Liefertermins im allgemeinen als nicht so gravierend eingeschätzt wie ein Qualitätsmangel (prototypische Bewertungen heißen hier Das kann schon mal vorkommen gegenüber Das darf nicht passieren), aber im speziellen Einzelfall reicht einem Kunden, der dringend auf das von ihm bestellte Produkt wartet, die lakonische, Normalität unterstellende Erklärung Wir haben eben zur Zeit Lieferschwierigkeiten nicht aus. Zudem ist eine solche Äußerung ohnehin unzweckmäßig, wenn Kunden dadurch den Eindruck gewinnen, Lieferschwierigkeiten seien in dem Unternehmen 'an der Tagesordnung'. Reklamationsbearbeiter sollten in solchen Fällen auf differierende Bewertungen von Kunden eingehen und versuchen, die Problemursachen für die Kunden genauer nachvollziehbar zu machen. Signalcharakter für aus Kundensicht gravierende Sachprobleme können Formulierungen wie in folgenden Beispielen haben.

K1: Einen [PRODUKTNAME] bestellt. Dann hat man mir gesagt am Montag, die wäre abgeschickt. Am Freitag sollte/ vergange- 
ner Woche sollte das gewesen sein. Jetzt frage ich mich nur, geht die jetzt über Rußland nach Deutschland?

K2: Ich brauch das Ding unbedingt. Meiner ist total kaputt. Da hat sich die Reparatur nicht mehr gelohnt. Und ich sitze da.

In der Mitarbeiterreaktion auf K2

M2: Ja leider haben wir gerade Lieferschwierigkeiten gerade mit dem [PRODUKTNAME]. Ich mach ihn jetzt eilig und dann ist er Anfang der nächsten Woche da, Frau Meier.

fehlt ein Eingehen auf die Situationsdarstellung von K2. Eine wenigstens indirekte Übernahme der Kundenperspektive ist in folgendem Beispiel sichtbar.

K3: Meine [PRODUKTTEIL] ist jetzt doch kaputtgegangen. Nun möchte ich gerne, daß die sofort geliefert wird ... Wann kann ich damit rechnen? Denn mein Ding, das explodiert fast. Und wir haben die Handwerker im Haus. Das ist das Problem.

M3: Ja ja dann ist es gut, daß sie uns nochmal angerufen haben. Ich kann die Sache dann noch ein bißchen schneller machen.

\subsubsection{Vorschnelle Problemtypisierung}

Mit Bewertungsdifferenzen ist auch folgender, häufig vorkommender Fall verbunden. Vor dem Hintergrund ihres Fach- und Institutionenwissens zeigen ReklamationsbearbeiterInnen oft die Tendenz, das von Kunden dargestellte Sachproblem in seinen Ursachen vorschnell zu typisieren, bevor eine gemeinsame Problemdefinition und -bewertung ratifiziert ist. Dies hat die Konsequenz, daß Kunden den Eindruck gewinnen, ihr Anliegen werde nicht als individuell relevantes Problem, sondern als trivialer Routinefall behandelt. Beispielsweise reagieren Kunden, die wegen eines verdorbenen Tiefkühlprodukts verärgert sind, unzufrieden, wenn ihnen als Problemerklärung die Routinekategorie 'Antauschaden' angeboten und dabei kein Bezug zu ihrem persönlichen Erleben hergestellt wird. ReklamationsbearbeiterInnen sollten sich deshalb zunächst mit Deutungen zurückhalten, der Problemdarstellung und Emotionsbekundung der Kunden möglichst offen zuhören sowie eine verbale Typisierung erst nach der gemeinsamen Problemratifikation vornehmen.

\subsubsection{Verharmlosung des Sachproblems}

Ausgesprochen 'allergisch' reagieren Kunden, wenn sie merken, daß ReklamationsbearbeiterInnen versuchen, das unbestreitbar vorliegende Sachproblem in seinen negativen Konsequenzen zu verharmlosen. 
M: Jaa, Frau [NAME], es ist folgendes eh Konservierungsstoffe und Farbstoffe sind nach dem deutschen Lebensmittel- und Bedarfsgegenständegesetz eh e zulassungspflichtich und eh die Konservierungsstoffe und Farbstoffe, die wir einsetzen, da können Sie von, da kann ich Sie beruhigen, sind wirklich harmlos, da passiert Ihnen gar nichts.

K: Also das kann so nicht sein. Ich habs also gelesen in Natur.

Wenn Mitarbeiterlnnen in solchen Fällen mit Hilfe von zweifelhaften Autoritätsargumenten auf ihrer Problemrückstufung insistieren, setzen sie ihre Glaubwürdigkeit aufs Spiel und riskieren, daß innen strategisches Verhalten zum Nachteil der Kunden unterstellt wird. Dabei ist zu bedenken, daß entsprechende negative Einstellungen auch dann vorhanden sein können, wenn sie von Kunden nicht explizit geäußert werden.

Auch bei der Klärung der Problemursachen treten in Reklamationsgesprächen oft typische, durch Beurteilungsdifferenzen bedingte Verständigungsschwierigkeiten auf. Wir wollen hier zwei auf die Klärung der Schuldfrage bezogene Problemtypen ansprechen.

\subsubsection{Vorschnelle Schuldabweisung}

In den von Fiehler und Kindt (1994) untersuchten Reklamationsgesprächen wurde beobachtet, daß ReklamationsbearbeiterInnen oft dazu neigen, von vornherein ein Verschulden des Unternehmens für das Problem ganz oder teilweise auszuschließen (sogenannte 100\%-Aussagen) oder die Verantwortlichkeit Dritter in den Vordergrund zu rücken. Ein Beispiel für die Kombination beider Verfahrensmöglichkeiten liefert die folgende Äußerung.

M: Also ich kann zu hundert Prozent garantieren, daß alle Produkte unseres Hauses die Firma im hundertprozentigen Zustand unser Haus verlassen, und auch auf dem Transportweg ist zu neunundneunzig Prozent gesichert, daß eh unsere Waren eh auch im guten Zustand bei dem Händler ankommen. Wie das natürlich vor Ort aussieht eh darauf haben wir leider keinen Einfluß. Es ist uns bewußt, daß in manchen Häusern eh die Gegebenheiten nicht unbedingt in Ordnung sind.

Das Verfahren der Schuldrelativierung oder -abwälzung wird von Kunden als Versuch, sich der Verantwortung zu entziehen, und als Zeichen von Inkooperativität der stärkeren Seite verstanden. Aussagen, die eine Schuld des Unternehmens ausschließen, werden überdies oft als implizite Schuldzuweisung an die reklamierende Person verstanden (nach dem Motto: Wenn wir es nicht waren, mußt du es gewesen sein). 
4.1.7 Differierende Vorstellungen über die Verantwortlichkeit für eine Problemlösung

Auch bei geklärter Schuldfrage gibt es häufig unterschiedliche Vorstellungen bei Kunde und Unternehmen hinsichtlich der Verantwortlichkeit für eine Problemlösung. Wir wollen dies wieder am Problem von Lieferschwierigkeiten demonstrieren. Das von Unternehmen zunehmend praktizierte 'just in time'-Prinzip führt zum Verzicht auf eine kostenaufwendige Lagerhaltung, ist aber sehr störungsanfällig. Hierfür haben Kunden, denen ein bestimmter Liefertermin zugesagt wurde, mit Recht kein Verständnis. Dies zeigt auch der folgende Dialog, in dem ein Kunde, der seit zwei Wochen auf ein bestelltes Ersatzteil wartet, zunehmend ungeduldiger wird.

M: Aber durch die Lieferschwierigkeiten ist das also immer noch nicht rausgegangen. Wir sind da echt in der Klemme, ne. Es ist also nicht so, daß wir da jetzt nicht liefern wollen. Wir können jetzt nicht.

K: Die ja warum haben sie die nicht am am Lager oder muß die erst produziert werden?

M: Wir ham kein Lager wir ham kein La/ Lager, ne.

K: Und wenn die bestellt wird, wird die erst produziert?

M: Die/ wir produzieren also praktisch so wie es k/ wie es kommt, ne, also praktisch aus der letzten Serienanfertigung die Sachen. Die gehen auch raus. Wir produzieren nicht auf Halde.

K: Das kann ja noch Monate dauern.

M: Nein ganz bestimmt nich.

K: Sagen sie nicht nein, Frau [NAME]. Ich habe die Nase voll. Jetzt jetzt reichts langsam.

Statt unmittelbar auf die Verärgerung des Kunden einzugehen, macht die Mitarbeiterin den Vorschlag, der Kunde möge der Firma eine Nachfrist setzen und auf diese Weise ggf. aus dem Kaufvertrag aussteigen. Diesen Vorschlag interpretiert der Kunde, dessen Gerät ohne das bestellte Ersatzteil wertlos wird, als Rückzug aus der Verantwortlichkeit des Unternehmens für eine angemessene Problemlösung.

K: Ach so. So so gut sind Sie schon dran, daß Sie es gar nicht mehr nötig haben, die Sachen zu äh 
4.2 Kommunikationsprobleme aufgrund unterschiedlicher Vorstellungen von der Reklamationsbearbeitung

Während der Bearbeitung der Aufgaben des Handlungsschemas für Reklamationsgespräche können bei Kunden und Reklamationsbearbeiterlnnen jeweils unterschiedliche Vorstellungen darüber bestehen, welche kommunikative Aktivität als nächster Handlungsschritt notwendig ist und in welcher Weise diese Aktivität durchgeführt werden sollte. Nachfolgend wollen wir vier verschiedene, häufig vorkommende Arten solcher Relevanzprobleme behandeln (vgl. auch Kindt 1998).

\subsubsection{Fehlende oder verzögerte Emotionsbearbeitung}

Ein erstes, besonders frappierendes Beispiel von Relevanzproblemen in Reklamationsgesprächen hat Antos (1989) beschrieben. In diesem Beispiel sind für Kunden und Mitarbeiterln zu Beginn des Gesprächs unterschiedliche kommunikative Aktivitäten relevant: Der Kunde möchte seine negativen Emotionen über das Sachproblem bearbeitet sehen, der Mitarbeiter aber will zuerst den Kunden anhand seiner Kundennummer identifizieren, um alle vorhandenen Informationen auf dem Bildschirm präsent zu haben. Prototypisch demonstriert folgendes, aus den Daten von Antos stammende Äußerungspaar eine solche Kommunikationsstörung:

K: Das ist eine furchtbare Schlamperei. Und ich verlange, daß der Vertreter das Gesamte bezahlen muß. Das verlang ich. Und wenn nicht, dann gehe ich zu meinem Rechtsanwalt. Ich bin schon seit 1960 so lange sind sie noch nicht bei (Firma X) Kunde und habe alles von (Firma X). Darf sowas nicht passieren.

M: Könnten Sie mir mal die Postleitzahl sagen von (Stadt Y) (...). Sie können weitersprechen Frau F selbstverständlich.

Generell sollte die Bearbeitung von Emotionen in der Kommunikation Vorrang vor anderen Aktivitäten haben. Wenn aber das Eingehen auf Emotionen zugunsten der Kundenidentifizierung zurückgestellt wird, muß dies zumindest explizit begründet werden. Eine solche Begründung wird manchmal in folgender Weise formuliert.

M1: Können Sie einen Augenblick in der Leitung bleiben. Ich schau mir den Vorgang mal an.

M2: Könnten Sie mir wohl einen Gefallen tun? Haben Sie die Rechnung vorliegen? Da müßte eine Bearbeitungsnummer gegeben sein.

Grundsätzlich erweist sich das Verfahren der betreffenden Firma, die Nennung der Bearbeitungsnummer zu verlangen, als kundenunfreundlich, weil Kunden die gewünschte Nummer oft nicht unmittelbar parat haben und dann langwierige (die Telefonkosten erhöhende) Suchprozeduren beginnen. Leider ist es dabei nicht die Regel, daß Suchhilfen wie die folgenden gegeben werden. 
M3: Können Sie mir daraus die Auftragsnummer geben? Auftragsnummer in dem grünen Kästchen rechts in dem grünen Kästchen.

Daß emotionale Kundenäußerungen übergangen werden, ist allerdings - wie wir schon am Gespräch aus Abschnitt 2 diskutiert haben - ein generelles Problem von Reklamationsgesprächen, das in den von uns untersuchten Aufnahmen immer wieder vorkommt und eine erfolgreiche Kommunikation maßgeblich erschwert. MitarbeiterInnen mögen vielleicht denken, daß es unzweckmäßig sei, sich selbst auf eine emotionale Ebene des Gesprächs einzulassen. In Wirklichkeit blockiert aber eine Nichtbearbeitung von Emotionen das weitere Gespräch, weil Kunden im weiteren Verlauf ihre emotionalen Reaktionen ständig wiederholen, solange sie nicht zumindest minimal beantwortet sind. Diese negative Konsequenz wird auch im nächsten Beispiel deutlich werden.

\subsubsection{Zyklische Gesprächsstrukturen}

Die Nichtbehandlung von Emotionen bildet zunächst ein lokal auftretendes Problem bei der Reklamationsbearbeitung. Gesprächskooperation und Verständigung werden aber auch durch globalere Relevanzprobleme nachhaltig behindert. In institutio-nellen Kontexten, in denen es um die Lösung von Problemen geht, treffen oft divergierende kommunikative Bearbeitungsformen von Institutionenvertretern einerseits und Kunden/Klienten andererseits aufeinander. Während die Institutionenvertreter im allgemeinen versuchen, nach einem emotionsfreien, rationalen Problemlösungsschema vorzugehen, greifen Kunden/Klienten oft auf eine in der Alltagskommunikation übliche Form der Problemdiskussion zurück. Diese beiden Kommunikationstypen sind nur schwer miteinander verbindbar, und deshalb kann es zur Entstehung ineffizienter, zyklischer Gesprächsstrukturen kommen, in denen die Beteiligten dem Prinzip der Selbstanknüpfung statt einer Fremdanknüpfung (vgl. Kindt 1985) folgen und sich zu aufwendigen Äußerungswiederholungen veranlaßt sehen. Hierzu ein Beispiel.

K: Ja dann hat man mich echt übertölpelt [. .] Also je/ das läuft, das läuft natürlich nicht.

M: Machen Se folgendes, Frau [Name], schicken Se uns das Schreiben zurück, was sie jetzt bekommen haben, ja, mit dem Vermerk, was sie mir gerade gesagt haben, wie sie meinen, daß das gelaufen ist, und dann klären wir die sache über unseren Außendienst ab.

K: Ne, also ich b/ ich bin wirklich dreißig Jahre Kunde bei Ihnen, und äh so was ist mir noch nicht passiert.

M: Hm ja, müssen wir abklären, wies zu der Bestellung gekommen ist. 
K: Ich habe ihm gesagt, es ist möglich, daß ich äh im April eventuell, man weiß ja nie, wie wie Finanzen stehen, ja, da hab ich gesagt, er möchte noch mal vorbeikommen. Dann war ein anderer Mitarbeiter von Ihnen hier, und da hab ich ihm das auch noch mal gesagt, also es klappt nicht im April, da meine Tochter Abitur macht und äh ja, da muß man auch ein bißchen was dazusteuern, und und und ich sag, das klappt nicht, ja ich sage das meinem Kollegen.

M: Ja, schreiben Sie das bitte so drauf auf das Schreiben, und wir klären das ab.

Der Mitarbeiter geht in seiner ersten hier zitierten Äußerung bereits zur Problemlösung über und kommt nicht der Notwendigkeit nach, den Betrugsvorwurf der Kundin emotiv zu bewerten. Dieses Verhalten entspricht zwar der gängigen Strategie von Reklamationsbearbeitern, keine affektiven Urteile vor einer genauen Sachverhaltsklärung abzugeben. Es besteht aber durchaus die Möglichkeit, auf Kundenemotionen mit einer sympathetischen Reaktion einzugehen (z.B. Ich kann gut verstehen, daß Sie erregt sind, Frau [Name], und deshalb schlage ich vor), ohne daß ein abschließendes Urteil über den Sachverhalt gefällt wird. Weil im konkreten Beispiel eine solche Reaktion des Mitarbeiters fehlt, sieht sich die Kundin in ihrer zweiten Äußerung veranlaßt, nicht auf den Verfahrensvorschlag des Mitarbeiters einzugehen, sondern zunächst selbst eine Problemevaluation abzugeben, auf die hin sie sich natürlich eine adäquate Reaktion des Mitarbeiters wünscht. Der Mitarbeiter aber knüpft nicht an ihre, sondern an seine eigene vorherige Äußerung an und wiederholt deshalb die letzte Position seines Verfahrensvorschlags. Das fehlende Eingehen auf ihre Problembewertung kann die Kundin nur so interpretieren, daß dem Mitarbeiter das zugrundeliegende Sachproblem noch nicht hinreichend klar ist, und deshalb kehrt sie in die Phase der Problemdarstellung mit einer erzählerischen Geschehensrekonstruktion zurück. Diese Darstellung wiederholt aus Kontinuitätsgründen der Erzählform wesentliche Geschehensbestandteile, die sie auch schon zu Beginn des Gesprächs vorgetragen hat. Zugleich expandiert sie ihre Darstellung aber um Bestandteile, aus denen hervorgeht, warum sie eine Bestellung nur unter Vorbehalt formulierte und wie sie ihre Bestellungsankündigung zurückgenommen hat. Diese Sachverhaltsdarstellung gibt dem Mitarbeiter die Gelegenheit, ein drittes Mal seinen Verfahrensvorschlag zu formulieren, und anschließend geht die Kundin auch auf diesen Vorschlag ein, so daß das Gespräch beendet werden kann.

\subsubsection{Kunden erzählen}

Neben der zyklischen Gesprächsstruktur zeigt das Beispiel von 4.2.2 noch ein anderes Relevanzproblem auf. Kunden bestimmter sozialer Gruppen geben Problemdarstellungen - wie im Alltag gewohnt - häufig in Form von Erzählungen, die aus der Sicht einer am Problemlösungsschema orientierten Kommunikation als zu ausführlich und deshalb als ineffizient erscheinen (vgl. Kindt 1993). Insofern ist es nicht verwunderlich, daß Reklamationsbearbeiterlnnen, die gefragt werden, welche 
Verhaltensweisen von Kunden sie für problematisch halten, u.a. angeben, Kunden würden häufig "ihre Lebensgeschichte erzählen". Diese Einschätzung beruht in zweifacher Weise auf einer sehr subjektiven Wahrnehmung. Einerseits ist MitarbeiterInnen nicht bewußt, daß sie in Fällen wie dem Beispiel von 4.2.2 selbst unnötigerweise ausführliches Erzählen und zyklische Problemdarstellungen provozieren. Andererseits kommt der Fall "Lebensgeschichte erzählen" zumindest in den von uns untersuchten Gesprächen nur sehr selten vor; trotzdem hat er für ReklamationsbearbeiterInnen eine besondere Bedeutung, weil sie sich im allgemeinen unsicher sind, ob und wie man den Erzählfluß entsprechender Kunden bremsen soll.

\subsubsection{Differierende Vorstellungen über die Ausführlichkeit der Ursachenklärung}

Das von Unternehmensangehörigen praktizierte Verfahren, das dargestellte Sachproblem umfassend aufzuklären, bevor Urteile abgegeben und Konsequenzen formuliert werden, wird von Kunden nicht gleichermaßen als relevant angesehen und kann deshalb zu erheblichen Mißverständnissen führen. Wenn dieses Verfahren nämlich nicht vor dem Kunden explizit als zweckmäßig legitimiert wird, dann sehen sie in ihm häufig einen Versuch des Unternehmens, die Verantwortung für das Sachproblem abzuschieben. Mißtrauen von Kunden entsteht z.B. dann, wenn ihnen - wie in folgendem Fall - eine schriftliche Sachverhaltsdarstellung abverlangt wird, die für sie einen erheblichen Aufwand bedeutet und die Problemlösung - aus ihrer Sicht - unnötig verzögert.

M: Schreiben Se das so in Ihrem Brief, mit den Beschwerden dabei, und/

K: (unterbricht) Ja, ich möchte das gleich zurückgeben. Ich möchte das los sein und Sie überweisen mir mein Geld wieder.

M: Ja, wir müssen das vorher abklären. Wir können nicht einfach das Gerät so zurücknehmen.

K: Wie/ wie/ wieso nich?

M: Weil da kein Mangel an dem Gerät dran ist!

K: Ja, das Gerät das/ das arbeitet doch nich wie es/ wie es äh äh äh beschrieben ist. Das/ das Ge/ das ist doch halber Kram ist doch das! ... (später:) ... und sie wollen mir hier nun sowas erzählen, ich äh soll da erst $n$ langen Schriftverkehr machen.

Eine Begründung für einen detaillierten Aufklärungsprozeß zu geben ist schon deshalb notwendig, weil Kunden weniger daran als an einer schnellen Bearbeitung ihrer Reklamation interessiert sind. 


\subsection{Kommunikationsprobleme aufgrund unterschiedlicher Interessen und Wissens- voraussetzungen}

Mit dem zuletzt in 4.2.4 behandelten Beispiel gelangen wir zu dem dritten und generellsten Typ von Problemursachen, der in jeder Art von Kommunikation zu berücksichtigen ist. Auch wenn vom Handlungsschema für Reklamationsgespräche her eine Klärung der Problemursachen vorgesehen ist, kann das individuelle Interesse der Gesprächspartner an einer solchen Klärung unterschiedlich groß sein. Ähnliches gilt für sämtliche Aufgaben des Handlungsschemas und mögliche thematischen Komplexe des Gesprächs. Dabei ist für Reklamationsbearbeiterlnnen wichtig, sich klarzumachen, daß auch das Interesse von Kunden an der Diskussion eines Sachverhalts vom Stand der Reklamationsbearbeitung abhängt. Wenn beispielsweise ein Kunde auf einer expliziten Schuldzuweisung für das Unternehmen insistiert, dann tut er dies häufig nur deshalb, weil ihm noch keine angemessene Problemlösung signalisiert wurde. Sobald er aber davon überzeugt ist, daß seine Reklamation erfolgreich sein wird, sieht er evtl. keinen Anlaß mehr, das Gespräch durch ausführliche Schuldzuweisungen zu verlängern.

Divergierende Interessen und unterschiedliche Kenntnisse können sich auf vielfältige Weise negativ auf Reklamationsgespräche auswirken, und die betreffenden Probleme lassen sich hier nicht vollständig darstellen. Deshalb wollen wir nur exemplarisch zwei wichtige Problemtypen ansprechen.

\subsubsection{Kundendrohungen}

Ein von ReklamationsbearbeiterInnen häufig genanntes Problem ist die Frage, wie man mit Kundendrohungen umgehen soll. Eine Drohung ist nur so lange funktional, als Kunden glauben, daß ihr Interesse an einer erfolgreichen Reklamation nicht auf andere Weise durchsetzbar ist. Wenn also eine nicht aufhebbare Interessendivergenz vorliegt, dann ist es für ReklamationsbearbeiterInnen wichtig zu wissen, welche Reaktionsmöglichkeiten sie haben. Einerseits kann ihnen nicht an einer Eskalation gelegen sein. Andererseits müssen sie im Sinne des generellen Verständigungsprinzips einer wechselseitigen Perspektivenübernahme versuchen, um Verständnis für das Unternehmensinteresse zu werben. Hierzu ein Beispiel.

Eine Kundin hat ein neues Gerät gekauft; dies scheint fehlerhaft; der Bearbeiter will ihr gratis den Kundendienst zwecks Prüfung und Reparatur vorbeischicken. Die Kundin möchte aber ein neues, kein repariertes Gerät für ihr Geld. Sie stellt diese Forderung in einem Rückruf, nachdem sie sich eine Stunde zuvor zunächst mit der Kundendienstlösung einverstanden erklärt hatte.

K: [...] und wenn die repariert wird, zahl ich keine hundertfünfzig Mark. Ich möchte ne neue Maschine. [...] Und wenn dat nich/ wenn dat nich passiert, hab ich grad schon der Dame gesagt, dann meld ich mich bei "Wie bitte" bei RTL, dat is mir scheißejal.

M: Ja. Sie brauchen uns aber wirklich nicht zu drohen hier, Frau [NAME], ich mein, das bringt uns ja nicht weiter. [...] 
Sie müssen uns die Möglichkeit geben, Sie sagen jetzt, die Maschine is kaputt. Das kann ich von hier aus aber gar nicht beurteilen ...

Der Mitarbeiter versucht hier, die Kundin von der Unzweckmäßigkeit ihrer Drohung zu überzeugen und ihr Verständnis für die vorgeschlagene Prüfung durch den Kundendienst zu erreichen. Je nach Situation können aber sehr unterschiedliche Strategien gegenüber Kundendrohungen angemessen sein.

\subsubsection{Fachsprachenbenutzung}

Neben divergierenden Interessen bilden immer wieder unterschiedliche Wissensvoraussetzungen von Kunden und MitarbeiterInnen eine wesentliche Ursache für Verständigungsprobleme. Eigentlich sollte man denken, daß dies den Gesprächsteilnehmern hinlänglich bewußt ist. Tatsächlich sind aber Personen, die spezielle Kenntnisse und Kommunikationsgewohnheiten erworben haben, im allgemeinen nicht ohne weiteres in der Lage, sich in die Perspektive anderer Teilnehmer hineinzuversetzen. Dies gilt auch für Reklamationsbearbeiterlnnen, die oft viel zu detailliert und in unangemessener Fachterminologie Sachverhalte darstellen, die für Kunden evtl. nur schwer nachvollziehbar sind. Hierzu ein Beispiel.

Ein Käufer hatte sich in einer mündlichen Absprache verpflichtet, bei einer Werbemaßnahme mit Produktpräsentation mitzuwirken, und hatte dafür einen Preisnachlaß für die erworbenen Geräte erhalten. Die Geräte wurden anschließend von ihm weiterverschenkt. Der Anrufer ist der Beschenkte, der von der Firma zur Mitwirkung bei der Produktpräsentation aufgefordert wird und sich dagegen wehrt.

M: Äh, wenn Sie natürlich der Beschenkte sind und nicht unser Vertragspartner, dann äh kann natürlich kein Anspruch erhoben werden, daß äh Sie eine Vorführung machen, das ist richtig. Nur muß eben äh äh dieser Anspruch auch wieder an den Vertragspartner zurückgegeben werden.

Der in dieser Äußerung verwendete entpersonalisierte juristische Fachjargon ist gegenüber alltagssprachlich formulierenden Kunden natürlich absolut unangemessen.

\section{Konsequenzen für die Durchführung von Kommunikationstrainings}

Die große Palette der in Abschnitt 4 diskutierten Kommunikationsprobleme sollte deutlich gemacht haben, daß es sich lohnt, Firmenmitarbeiterlnnen vor ihrem Einsatz in Reklamationsgesprächen auf das Auftreten und die Lösung solcher Probleme vorzubereiten. Dies kann nach unseren Erfahrungen besonders gut gelingen, wenn die Mitarbeiterlnnen in Kommunikationstrainings mit (möglichst) authentischen Aufzeichnungen und Transkriptionen von Reklamationsgesprächen konfrontiert werden, die in der entsprechenden Firma selbst geführt wurden oder 
den dortigen Gegebenheiten vergleichbar sind. Als konkrete Lernziele müssen solche Trainings u.a. folgende drei Punkte umfassen. Die Mitarbeiterlnnen sollten

- die kommunikativen Aufgaben des in Abschnitt 3 dargestellten Handlungsschemas kennen und zugleich typische sprachliche Realisierungen der Aufgabendurchführung beherrschen;

- auf der Basis des Handlungsschemas in der Lage sein, in realen Reklamationsgesprächen die jeweils vorkommenden Kommunikationsprobleme zu identifizieren und korrekt zu typisieren;

- ihren Handlungsspielraum für Lösungsmöglichkeiten der jeweiligen Problemtypen kennen, um in konkreten Gesprächen situationsangemessen reagieren zu können.

Die genauere Konzeption eines solchen Trainings wird in Fiehler (1997) vorgestellt. Ausführungen über methodische Vorgehensweises in solchen Trainings sind an anderer Stelle in diesem Band zu finden (vgl. Lepschy und Becker-Mrotzek/Brünner in Bd. 2). Betont sei hier aber, daß Lernerfolge in Trainings für Reklamationsgespräche nur erwartbar sind, wenn die zu vermittelnden Kommunikationsstrategien jeweils auf Aufgabenschritte im Handlungsschema bezogen und durch sprachliche Formulierungen operationalisiert werden, deren Zweckmäßigkeit empirisch abgesichert ist.

Der Vorteil diskursanalytisch fundierter Trainings liegt dann einerseits darin, daß keine pauschalen, sondern für spezifische Kontexte definierte Handlungsempfehlungen gegeben werden, die zumindest für Standardfälle ausreichen. Und da andererseits nicht nur jeweils eine bestimmte Handlung vorgeschrieben, sondern verschiedene Möglichkeiten vorgeschlagen werden, bleibt den Teilnehmerlnnen die Entscheidung darüber vorbehalten, welche der Handlungsalternativen je nach eigenem Kommunikationsstil und nach speziellen Situationsbedingungen als angemessen ausgewählt wird. In diesem differenzierten Sinne läßt sich behaupten, daß man lernen kann, Kommunikationsprobleme in Reklamationsgesprächen zu vermeiden oder zu minimieren.

\section{Literatur}

Antos, G. (1989). Kontraproduktive Gespräche. Zur Diskrepanz zwischen Musterwissen und interaktioneller Durchführung. In: Weigand, E. \& Hundsnurscher, F. (Hrsg.), Dialoganalyse II. Referate der 2. Arbeitstagung, Bochum 1988. Bd. 2. Tübingen: Niemeyer, 253-263.

Bruhn, M. (1982). Konsumentenzufriedenheit und Beschwerden. Erklärungsansätze und Ergebnisse einer empirischen Untersuchung in ausgewäh/ten Konsumbereichen. Frankfurt a.M/Bern: Lang.

Ehlich, K. \& Rehbein, J. (1976). Halbinterpretative Arbeitstranskriptionen (HIAT). In: Linguistische Berichte 45, 21-41.

Fiehler, R. (1990). Kommunikation und Emotion. Theoretische und empirische Untersuchungen zur Rolle von Emotionen in der verbalen Interaktion. Berlin: de Gruyter 1990.

Fiehler, R. (1997). Training: Optimierung persönlicher und telefonischer Reklamationsgespräche. In: Obermann, C. \& Schiel, F. (Hrsg.), Trainingspraxis. Köln: Bachem, 39-56.

Fiehler, R. \& Kindt, W. (1994). Reklamationsgespräche. Schulungsperspektiven auf der Basis von Ergebnissen diskursanalytischer Untersuchungen. In: Bartsch, E. (Hrsg.), Sprechen, Führen, 
Kooperieren in Betrieb und Verwaltung. Kommunikation in Unternehmen. München, Basel: Reinhardt, 255-269.

Hansen, U. \& Schoenheit, I. (Hrsg.)(1985). Verbraucherabteilungen in privaten und öffentlichen Unternehmen. Frankfurt a.M., New York: Campus.

Hansen, U. \& Schoenheit, I. (Hrsg.)(1987). Verbraucherzufriedenheit und Beschwerdeverhalten. Frankfurt a.M., New York: Campus.

Kallmeyer, W. (1985). Handlungskonstitution im Gespräch. Dupont und sein Experte führen ein Beratungsgespräch. In: Gülich, E. \& Kotschi, Th. (Hrsg.), Grammatik, Konversation, Interaktion. Beiträge zum Romanistentag 1983. Tübingen: Niemeyer, 81-122.

Kindt, W. (1985). Selbstanknüpfung und Stereotypie in Politikeräußerungen. In: Sucharowski, W. (Hrsg.), Gesprächsforschung im Vergleich. Analysen zur Bonner Runde nach der Hessenwahl 1982. Tübingen: Niemeyer, 146-176.

Kindt, W. (1993). Struktur, Funktion und Dynamik von Erzählungen. In: Janota, J. (Hrsg.), Kultureller Wandel und die Germanistik in der Bundesrepublik. Bd. 1. Vielfalt der kulturellen Systeme und Stile. Vorträge des Augsburger Germanistentags 1991. Tübingen: Niemeyer, 151-166.

Kindt, W. (1998). Konzeptuelle Grundlagen einer Theorie der Verständigungsprobleme. In: Fiehler, R. (Hrsg.), Verständigungsprobleme und gestörte Kommunikation. Opladen: Westdeutscher Verlag, 17-43.

Kindt, W. \& Weingarten, R. (1984). Verständigungsprobleme. In: Deutsche Sprache 3, 193-218.

Riemer, M. (1986). Beschwerdemanagement. Frankfurt a.M., New York: Campus.

Schnieders, G. (i.D.). Authentische und simulierte Reklamationsgespräche - ein exemplarischer Vergleich. Erscheint in: Rehbein, J. (Hrsg.), Spektrum der Funktionalen Pragmatik. 